\title{
Concept-Based Information Retrieval Using Explicit Semantic Analysis
}

\author{
OFER EGOZI, SHAUL MARKOVITCH, and EVGENIY GABRILOVICH, \\ Technion-Israel Institute of Technology
}

Information retrieval systems traditionally rely on textual keywords to index and retrieve documents. Keyword-based retrieval may return inaccurate and incomplete results when different keywords are used to describe the same concept in the documents and in the queries. Furthermore, the relationship between these related keywords may be semantic rather than syntactic, and capturing it thus requires access to comprehensive human world knowledge. Concept-based retrieval methods have attempted to tackle these difficulties by using manually built thesauri, by relying on term cooccurrence data, or by extracting latent word relationships and concepts from a corpus. In this article we introduce a new concept-based retrieval approach based on Explicit Semantic Analysis (ESA), a recently proposed method that augments keywordbased text representation with concept-based features, automatically extracted from massive human knowledge repositories such as Wikipedia. Our approach generates new text features automatically, and we have found that high-quality feature selection becomes crucial in this setting to make the retrieval more focused. However, due to the lack of labeled data, traditional feature selection methods cannot be used, hence we propose new methods that use self-generated labeled training data. The resulting system is evaluated on several TREC datasets, showing superior performance over previous state-of-the-art results.

Categories and Subject Descriptors: H.3.1 [Information Storage and Retrieval]: Content Analysis and Indexing; H.3.3 [Information Storage and Retrieval]: Information Search and Retrieval-Query formulation; relevance feedback

General Terms: Algorithms, Experimentation

Additional Key Words and Phrases: Concept-based retrieval, explicit semantic analysis, feature selection, semantic search

ACM Reference Format:

Egozi, O., Markovitch, S., and Gabrilovich, E. 2011. Concept-Based information retrieval using explicit semantic analysis. ACM Trans. Inf. Syst. 29, 2, Article 8 (April 2011), 34 pages.

DOI $=10.1145 / 1961209.1961211 \mathrm{http} / / /$ doi.acm.org/10.1145/1961209.1961211

\section{INTRODUCTION}

Information Retrieval (IR) systems aim at providing the most relevant documents to a user's query. Early IR systems were primarily used by retrieval experts, hence initial IR methodology was based on keywords manually assigned to documents, and on complicated Boolean queries. As automatic indexing and natural language queries gained popularity in the 1970's, IR systems became increasingly more accessible to nonexpert

This article extends the authors' previous work, titled "Concept-Based Feature Generation and Selection for Information Retrieval," which appeared in the 23rd AAAI Conference on Artificial Intelligence.

Authors' addresses: O. Egozi, S. Markovitch, Department of Computer Science, The Technion, Haifa, 32000, Israel; email: \{ofere, shaulm\}@cs.technion.ac.il; E. Gabrilovich, Yahoo! Research, 4301 Great America Parkway, Santa Clara, CA 95054; email: gabr@yahoo-inc.com.

Permission to make digital or hard copies of part or all of this work for personal or classroom use is granted without fee provided that copies are not made or distributed for profit or commercial advantage and that copies show this notice on the first page or initial screen of a display along with the full citation. Copyrights for components of this work owned by others than ACM must be honored. Abstracting with credit is permitted. To copy otherwise, to republish, to post on servers, to redistribute to lists, or to use any component of this work in other works requires prior specific permission and/or a fee. Permission may be requested from Publications Dept., ACM, Inc., 2 Penn Plaza, Suite 701, New York, NY 10121-0701, USA, fax +1 (212) 869-0481, or permissions@acm.org.

(C) 2011 ACM 1046-8188/2011/04-ART8 $\$ 10.00$

DOI 10.1145/1961209.1961211 http://doi.acm.org/10.1145/1961209.1961211 
users. Documents were indexed by automatically considering all terms in them as independent keywords, in what is known as the Bag-of-Words (BOW) representation, and query formatting was simplified to a short natural language formulation. However, even as the keywords became "noisier," the basic methodology for indexing them remained unchanged. Thus, these nonexpert users were increasingly faced with what was described as "the vocabulary problem" [Furnas et al. 1987]. The keywords chosen by users were often different from those used by the authors of the relevant documents, lowering the systems' recall rates. In other cases, the contextual differences between ambiguous keywords were overlooked by the BOW approach, reducing the precision of the results. These two problems are commonly referred to as synonymy and polysemy, respectively.

IR researchers attempted to resolve the synonymy problem by expanding the original query with synonyms of query keywords [Voorhees 1994]. However, the relationship between the keywords chosen by users and those used by authors often extends beyond simple synonymy. Consider the short query "Estonia economy," an actual query (\#434) in the TREC-8 Adhoc test collection [Voorhees and Harman 1999]. A relevant document may discuss announcements by the ministry of trade in Tallinn (the Estonian capital), with no mention of any direct synonym of any of the query keywords.

To handle such problems, new query expansion methods that rely on corpus-based evidence were suggested. For example, [Xu and Croft 2000] suggested identifying terms that cooccur with query keywords in the top-ranked documents for the query, to be used as expansion terms that are more broadly related to the query (such as "trade" and "Tallinn," in this example). Such approaches showed significant improvement, but require manual tuning in order not to adversely affect performance: too few expansion terms may have no impact, and too many will cause a query drift [Mitra et al. 1998].

To tackle polysemy, the main proposed method was to apply automatic word sense disambiguation algorithms to documents and query. Disambiguation methods use resources such as the Wordnet thesaurus [Voorhees 1993] or cooccurrence data [Schuetze and Pedersen 1995] to find the possible senses of a word and map word occurrences to the correct sense. These disambiguated senses are then used in indexing and in query processing, so that only documents that match the correct sense are retrieved. The inaccuracy of automatic disambiguation is the main obstacle in achieving significant improvement using these methods, as incorrect disambiguation is likely to harm performance rather than merely not improve it.

Concept-based information retrieval is an alternative IR approach that aims to tackle these problems differently. Concept-based IR represents both documents and queries using semantic concepts, instead of (or in addition to) keywords, and performs retrieval in that concept space. This approach holds the promise that representing documents and queries (or augmenting their BOW representation) using high-level concepts will result in a retrieval model that is less dependent on the specific terms used [Styltsvig 2006]. Such a model could yield matches even when the same notion is described by different terms in the query and target documents, thus alleviating the synonymy problem and increasing recall. Similarly, if the correct concepts are chosen for ambiguous words appearing in the query and in the documents, nonrelevant documents that were retrieved with the BOW approach could be eliminated from the results, thus alleviating the polysemy problem and increasing precision.

Existing concept-based methods can be characterized by the following three parameters.

(1) Concept representation: the "language" the concepts are based on. In this article we distinguish between approaches to concept-based IR that used explicit 
concepts, which represent real-life concepts resembling human perception [Gauch et al. 2003; Voorhees 1993], and approaches that utilized implicit concepts, generated by extracting latent relations between terms or calculating probabilities of encountering terms, that may not necessarily align with any human-interpretable concept [Deerwester et al. 1990; Hofmann 1999; Yi and Allan 2009].

(2) Mapping method: the mechanism that maps natural language texts to these concepts. The most accurate mechanism would likely be manual, building a handcrafted ontology of concepts with a list of words to be assigned to each [Miller et al. 1990], but such an approach involves significant effort and complexity. The mapping can also be automatic, using machine learning [Gauch et al. 2003], though this would usually imply less accurate mapping.

(3) Use in IR: the stages in which the concepts are used. Concepts would be best used throughout the entire process, in both indexing and retrieval stages [Gonzalo et al. 1998]. A simpler but less accurate solution would apply concept analysis in one stage only, as in concept-based query expansion over BOW retrieval [Grootjen and van der Weide 2006].

Of all the approaches suggested so far for concept-based IR, none fared well on all three characteristics just described. An ideal approach would use explicit semantic concept representation that is grounded in human cognition and intuitive to use and reason over, with no limits on domain coverage or conceptual granularity, would support a fully automatic mechanism for mapping texts onto those concepts, would be computationally feasible even for very large corpora, and would integrate conceptbased processing in both indexing and retrieval stages.

In this article we propose a novel concept-based IR approach that meets all of the preceding requirements, using Explicit Semantic Analysis (ESA) to augment the standard BOW representation. The concepts used are taken from a very comprehensive, human-defined ontology of explicit concepts. Text analysis methods are used to automatically and efficiently extract these concepts and represent any document or query text using them. Finally, the proposed system builds upon existing IR methodology and augments BOW representation with concepts in both indexing and retrieval, using standard data structures and ranking methods.

We show that a naive implementation of IR using these concepts is insufficient, due to the concepts' inherent noisy nature. We address these difficulties by embedding feature selection methods into the retrieval process, and then proceed to introduce the full system which uses these selected concepts to augment a standard keyword-based retrieval. We evaluate the proposed system on TREC datasets to show significant improvement in performance compared both with our own baseline and with published results of other state-of-the-art systems.

Our main contributions in this work are threefold: a framework for using the ESA representation method in information retrieval, a method for integrating feature selection into the concept-based IR task, and three selection methods that are based on common AI methods and shown beneficial for the task at hand.

The remainder of this article is organized as follows. Section 2 provides background on ESA. Sections 3 to 5 describe the proposed concept-based algorithms and empirical evaluation results. Section 6 surveys related work on concept-based IR, and Section 7 concludes.

\section{BACKGROUND}

Explicit Semantic Analysis, or ESA [Gabrilovich and Markovitch 2006], is a recently proposed method for semantic representation of general-domain natural language texts. ESA represents meaning in a high-dimensional space of concepts, automatically 


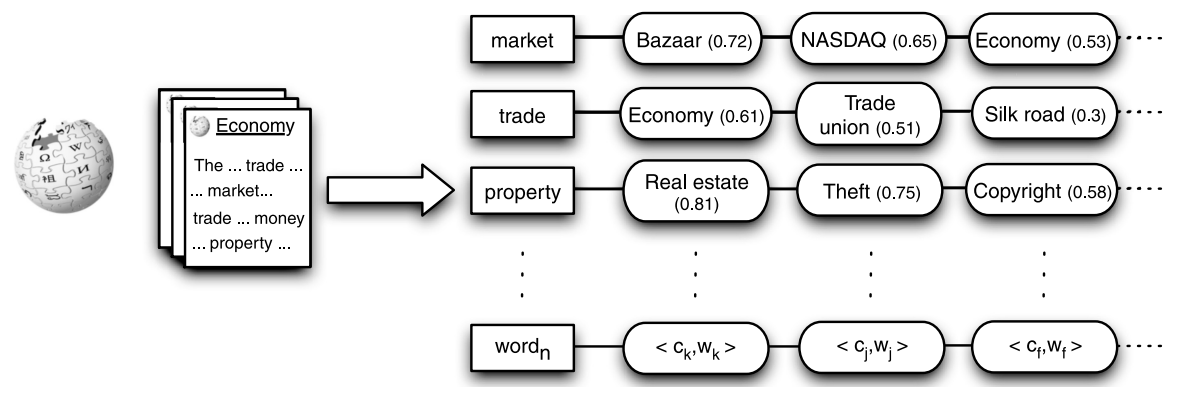

Fig. 1. Generation of an ESA model from Wikipedia articles. The articles and words in them are processed to build a weighted inverted index, representing each word as a vector in the space of all Wikipedia concepts (articles).

derived from large-scale human-built repositories such as Wikipedia. ${ }^{1}$ Since it was first proposed, ESA has been successfully applied to text categorization [Chang et al. 2008; Gabrilovich and Markovitch 2006; Gupta and Ratinov 2008], semantic relatedness calculation [Gabrilovich and Markovitch 2007; Gurevych et al. 2007], cross-language information retrieval [Potthast et al. 2008; Sorg and Cimiano 2008], and concept-based information retrieval [Egozi et al. 2008].

In Wikipedia-based ESA, the semantics of a given word are described by a vector storing the word's association strengths to Wikipedia-derived concepts. A concept is generated from a single Wikipedia article, and is represented as a vector of words that occur in this article weighted by their tf.idf score. Once these concept vectors are generated, an inverted index is created to map back from each word to the concepts it is associated with. Thus, each word appearing in the Wikipedia corpus can be seen as triggering each of the concepts it points to in the inverted index, with the attached weight representing the degree of association between that word and the concept. The process is illustrated in Figure 1.

As an example, these are the top ten concepts triggered by the word "investor": (1) Investment; (2) Angel investor; (3) Stock trader; (4) Mutual fund; (5) Margin (fiNANCE); (6) MOdERN PORTFOLIO THEORY; (7) EQUity INVESTMENT; (8) EXCHANGE-TRADED FUnd; (9) HEDGe FUnd; (10) PonZI SCheme. Even without reading the Wikipedia articles associated with these concepts, it will be intuitively clear to most readers that these concepts are relevant to the input word. The concepts' labels also exhibit a degree of semantic similarity and relatedness to the input term that extends simple synonymy. As a result, computing word relatedness between words based on their Wikipedia-ESA representation was shown more effective than any other current method [Gabrilovich and Markovitch 2007].

With this resource in hand, any input word to a text processing task can now be semantically represented as a sparse vector in the high-dimensional space of Wikipedia concepts. Larger text fragments are represented as a concept vector that is a combination of the separate vectors of its individual terms, and ESA operations can then be carried out by manipulating these vectors. For example, computing semantic relatedness between two texts can be reduced to generating the ESA concept vectors for each of them, and then calculating their cosine similarity.

To illustrate the nature of ESA concepts, we show the top concepts generated by our ESA implementation for two short news clip fragments.

\footnotetext{
${ }^{1} \mathrm{http}: / /$ www.wikipedia.org
} 
- Text. "A group of European-led astronomers has made a photograph of what appears to be a planet orbiting another star. If so, it would be the first confirmed picture of a world beyond our solar system."

Top generated concepts. (1) Planet; (2) Planetary orbit; (3) Solar system; (4) Extrasolar Planet; (5) Jupiter; (6) Astronomy; (7) Definition of Planet; (8) Pluto; (9) Minor Planet; (10) PSR 1257+12

All concepts are highly relevant and describe or relate to the subject of the text, with the fourth concept (EXTRASOLAR PLANET) being the exact topic, despite the fact that these words were not explicitly mentioned in the text. PSR $1257+12$ is the name of a pulsar around which the first extrasolar planets were discovered orbiting.

- Text. "New Jaguar model unveiled by firm"

Top generated concepts. (1) JAGUAR XJ; (2) JAGUAR (CAR); (3) ForD Motor CoMpanY; (4) Jaguar XK; (5) Land Rover Range Rover; (6) Jaguar S-Type; (7) Jaguar XTyPe; (8) Nissan Micra; (9) V8 Engine; (10) JAGUar E-TYPe

This example demonstrates the disambiguation power of ESA, as the top concepts all refer to Jaguar the car maker rather than to the namesake animal (e.g., the ESA concept JAGUAR) or American football team (e.g., the ESA concept JACKSONVILLE JAGUARS). Despite the text containing no explicit car-related terms, words such as "model" and "unveil" were more related to the industry meaning and helped trigger the correct concepts. The concepts generated also hint at rich world knowledge, such as the business relations to FORD Motor COMPANy and LAND Rover RANGE ROVER and the use of a V8 Engine on Jaguar models. The Nissan Micra concept was triggered by a Micra variant that was inspired by a Jaguar model.

We believe that the use of a knowledge repository as large and diverse as Wikipedia creates a powerful concept ontology, well suited for concept-based IR. Wikipedia's broad coverage of a huge range of topics, coupled with ESA's automatic ontologybuilding capability, yields a highly fine-grained ontology. In addition, the language coverage of the inverted index, mapping from a massive aggregation of natural language terms (the entire Wikipedia corpus) to the concepts in which they occur, produces a powerful classifier to automatically map any given text fragment to this concept ontology.

In this context we note an interesting work by [Anderka and Stein 2009], who hypothesized, and attempted to empirically show, that the nature of the text collection used to build ESA from (i.e., its structure or the semantics of its nodes) has much less impact on ESA performance than its size, by showing similar results with ESA models built on other types of text collections than Wikipedia. It should be noted, however, that the authors only experimented with a single application of ESA (text similarity), and used just a single, extremely small and homogenous test collection of 50 news documents (which may actually help explain how the best performing ESA base collection was also a news resource: Reuters).

Finally, we point out that building an ESA model based on a semantics-based ontology such as Wikipedia's, or in another implementation the Open Directory Project [Gabrilovich and Markovitch 2005], generates more meaningful and human-readable concepts that can provide additional reasoning for the researcher and for system users.

\section{ESA-BASED RETRIEVAL}

Given the described advantages of ESA as a semantic representation and its demonstrated success in other text analysis tasks, it appears well suited for building a successful concept-based IR model. In this section we introduce our first algorithm for concept-based IR using ESA representation. The algorithm maps documents and queries to the Wikipedia-ESA concept space, and performs indexing and retrieval in 
that space. We then evaluate the algorithm's performance on TREC datasets. We show that combining concept-based relevancy of documents with that of passages in these documents performs best for ESA-based retrieval. We also find that the quality of generated concepts is lower than expected, and analyze the potential causes and remedies to be applied in the next section.

\subsection{ESA Concept-Based Indexing}

We use ESA to map each document in the corpus to a weighted vector of concepts. Like BOW vectors, concept-based vectors are also sparse, with concept weights being zero for most of the Wikipedia concepts. Nevertheless, given that each word in the document to be indexed may still be related to a large number of concepts, and that a document containing a collection of words is likely to be related to an even larger number, indexing the entire list of related concepts for every document is not feasible. We therefore use only those concepts with the highest weights (association scores). In a sorted representation of the vector, this subset of concepts is simply its prefix.

Long documents are more difficult to map in full into the ESA concept space. A small part of a long document might be relevant to the current query, but the semantics of this part may be underrepresented in the concepts vector for the full document. A similar problem exists also in BOW approaches, where the Term Frequency (TF) measure must be normalized [Singhal et al. 1995] to account for documents of different lengths. However, for concept-based retrieval the challenge is even greater, because of the averaging effect of the representation of longer text fragments and the practical need to use only a small subset of the representation concepts. Concepts generated for a short section that is relevant to the query and is part of a larger document discussing nonrelevant topics might be pruned out of the indexed vector, since the concepts' weights in the overall document concepts vector might be too low to impact the retrieval results.

Previous research using BOW representation has shown that breaking long documents into shorter passages can improve document retrieval [Callan 1994; Liu and Croft 2002], with the ranking of passages viewed as evidence to the relevance of their source documents. Furthermore, it has often been shown that fixed-length passages yield better results than passages based on syntactic or semantic segmentation [Callan 1994; Kaszkiel and Zobel 2001]. We therefore suggest a similar approach, breaking documents into length-based overlapping passages and representing each passage separately by its own generated set of concepts. We expect such an approach to achieve better results, in particular with long documents that cover several themes.

Note that while [Gabrilovich and Markovitch 2006] also split documents into sentence and paragraph contexts in applying ESA to text categorization, they eventually combined the concepts of these subcontexts into a single unified representation. In our approach, each passage is indexed and may be retrieved as a stand-alone unit of information. Thus, a passage is ranked separately as an independent indicator of its original document's relevance.

We now have, for any document to be indexed, a set of passages and a concept vector representation for each. We index these concepts in a standard IR inverted index, using the concepts' unique identifiers as tokens. The score associated with each concept in the vector is used as the token weight, equivalent to term frequency in standard text indexing. The pseudocode for the preceding indexing algorithm is described in Figure 2.

\subsection{ESA-Based Retrieval Algorithm}

Upon receiving a query, our algorithm first converts it to an ESA concept vector. The representation method is identical to the one by which documents and passages are 


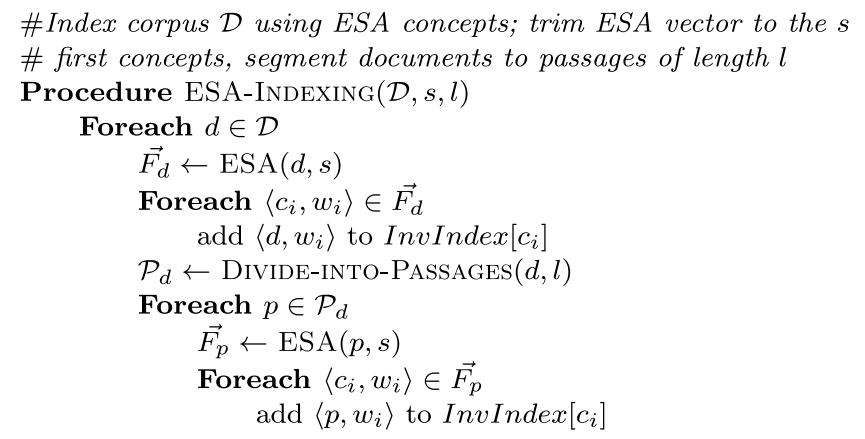

Fig. 2. ESA-based indexing in an inverted index.

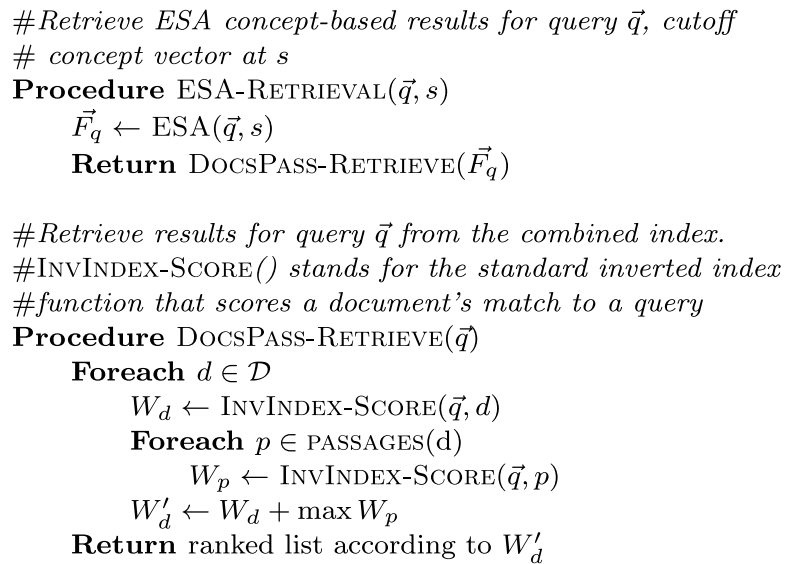

Fig. 3. ESA-based retrieval.

represented at index time. Having indexed full documents and passages, we now have to choose how these two types of evidence are to be combined for ranking. Following [Callan 1994], we retrieve both sets of results and sum each document's full score with the score of the best performing passage in it. ${ }^{2}$ The documents are then sorted by this combined score and the top scoring documents are output, as described ${ }^{3}$ in Figure 3.

The retrieval algorithm has a single parameter $s$ controlling the cutoff (as described in the previous section) of the query concept vector. The value for $s$ may be chosen to be the same as that in the indexing process, but not necessarily. Indexing the entire corpus with large cutoff values would incur significant storage and computation costs, and is therefore not feasible. The query representation, on the other hand, being derived from a much shorter text fragment and incurring no such costs, could benefit from a finer representation, using a higher value for $s$.

${ }^{2}$ We also experimented with assigning different weights to these two summed scores but found no improvement in doing so.

${ }^{3}$ In practice, the retrieval process is optimized to not iterate on all indexed documents; hence this combination is performed only for the top ranking documents (the top 1000 in our case), but the principle is similar. 


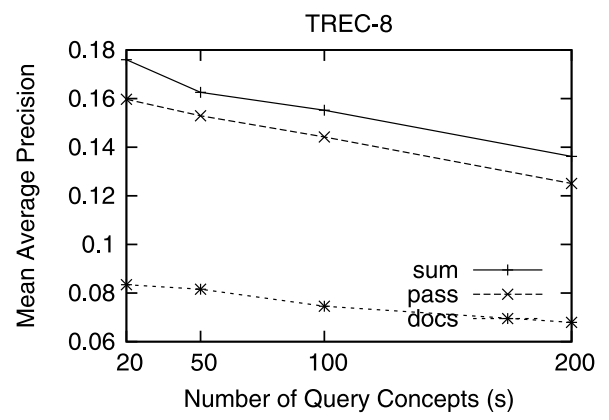

Fig. 4. ESA-based retrieval performance as a function of ESA cutoff and ranking contexts.

\subsection{Empirical Evaluation}

In order to evaluate the usefulness of ESA concept-based retrieval, we carried out a set of experiments.

3.3.1. Implementation. We used Xapian, ${ }^{4}$ an open-source probabilistic IR library, as the basis for our experimental platform. Document keywords and concepts were indexed in a Xapian inverted index. In addition, Xapian's implementation of the popular Okapi BM25 ranking formula [Robertson and Walker 1999] served as a BOW baseline. Most of the experiments used the TREC-8 Adhoc [Voorhees and Harman 1999] and the TREC Robust 2004 [Voorhees 2005] datasets. The TREC-8 dataset consists of 528,000 documents (mostly newswire) and 50 topics (information-need descriptions, to be transformed into queries), and the Robust-04 dataset uses the same document collection with a different set of 49 topics. We used only the short ("title") queries in TREC topics, since these short (1-3 words) queries better represent common reallife queries, in particular on the Web [Arampatzis and Kamps 2008]. In addition, short texts were shown to benefit most from conceptual representation [Gabrilovich and Markovitch 2006; Ozcan and Aslandogan 2005] as their extremely sparse BOW representation suffers most from the synonymy problem. We use the Mean Average Precision (MAP) evaluation measure, commonly used by TREC participants, which combines precision and recall while assigning higher importance to the higher-ranking relevant documents.

Documents and passages were stemmed, stopped, and indexed by their BOW representation, to be used by the BOW baseline method (in which we also combined passage and document ranking). Then, ESA-based representations were created and indexed separately as described in Figure 2. Passages were set to be fixed-size overlapping segments, shown most effective by [Kaszkiel and Zobel 2001], with passage size set to 50 words. We also tried to use longer passages (200 words) but this proved less effective.

The ESA implementation used in our experiments is as described in [Gabrilovich and Markovitch 2006], with ESA vector cutoff in the indexing stage ( $s$ in Figures 2 and 3 ) set to 50 concepts for practical reasons (index size). ${ }^{5}$

3.3.2. Results. Figure 4 shows the performance (MAP) of our ESA-based retrieval algorithm for various parameter values. To assess the impact of the concept vector

\footnotetext{
${ }^{4} \mathrm{http} / / /$ xapian.org/

${ }^{5}$ We have also experimented with indexing the 100 strongest concepts instead of the 50 strongest, and found no significant impact on the performance.
} 
truncation, we measured performance for varying values of $s$ (the ESA vector cutoff level) in the query vector. In addition, to validate the added value of combining documents and passages scores, we compared performance of the combined score to that of documents and passages alone.

As Figure 4 clearly shows, passage context outperforms document context significantly, but the best results are achieved when both are combined, an outcome that is consistent with previous IR findings for BOW representations [Croft 2000]. We will be using the combined documents+passages scoring from here onwards.

Results for increasing values of $s$ indicate that merely adding lower-ranking concepts in the ESA vector does not improve retrieval. Not only does the precisionoriented MAP score decrease as concepts are added, but the absolute recall (measured in the top 1000 retrieved documents) decreases as well. This finding suggests that some of the generated concepts may be detrimental, and that successful application of ESA to IR may require further selection of the concepts initially generated for the query. We will revisit this hypothesis later on.

However, even when choosing the best performing parameter values, ESA-based retrieval (MAP of 0.1760) is significantly inferior to that of our BOW baseline (MAP of 0.2481). Considering the superior results obtained when ESA-based representation was applied to previous text analysis applications [Gabrilovich and Markovitch 2006, 2007], this result is quite surprising, and we must further analyze it before proceeding to augment BOW with ESA concepts. In the following subsection, we conduct a qualitative analysis of specific retrieval cases in order to better understand the causes of this inferior retrieval and to suggest ways to remedy them.

3.3.3. Qualitative Analysis. The results show that ESA-based retrieval can indeed, as expected, identify relevant documents even when these do not include query terms or their simple synonyms. Let us consider TREC query 411 ("salvaging shipwreck treasure"). The following short relevant document was retrieved by the ESA-based method but not by the BOW baseline.

"ANCIENT ARTIFACTS FOUND. Divers have recovered artifacts lying underwater for more than 2,000 years in the wreck of a Roman ship that sank in the Gulf of Baratti, 12 miles off the island of Elba, newspapers reported Saturday."

The top-10 concepts generated for this document were the following.

SCUBA DIVING

WRECK DIVING

RMS TITANIC

USS HOEL (DD-533)

SHIPWRECK

UNDERWATER ARCHAEOLOGY

USS MAINE (ACR-1)

MARITIME ARCHAEOLOGY

TOMB RAIDER II

USS MEADE (DD-602)

The query's top-10 concepts were as follow.

SHIPWRECK

TREASURE

MARITIME ARCHAEOLOGY

MARINE SALVAGE

History OF THE BRITISH VIRGIN ISLANDS 
WRECKING (SHIPWRECK)

KEY WEST, FLORIDA

FLOTSAM AND JETSAM

WRECK DIVING

SPANISH TREASURE FLEET

With 3 matches in the top-10 concepts (and more in lower positions), the ESA-based method was capable of retrieving this relevant document as its third ranked result, despite the fact that not one of the query terms appears in the document's text.

Let us now examine a contrary example, where concept-based retrieval returned a nonrelevant document, one that was not returned by the BOW baseline. We revisit query 434 ("Estonia economy"), for which the following short document was retrieved using the concept-based method.

"Olympic News In Brief: Cycling win for Estonia. Erika Salumae won Estonia's first Olympic gold when retaining the women's cycling individual sprint title she won four years ago in Seoul as a Soviet athlete."

Although this document is Estonia-related, it concerns not economy but sports. The document's top-10 concepts were the following.

ESTONIA AT THE 2000 SUMMER OLYMPICS

ESTONIA AT THE 2004 Summer OlympiCS

2006 CommonWEALTH Games

Estonia AT THE 2006 Winter OLyMPICS

1992 SUMMER OLYMPICS

Athletics At the 2004 Summer Olympics - Women's Marathon

2000 SUMMER OLYMPICS

2006 WINTER OLYMPICS

CROSS-COUNTRY SKIING AT THE 2006 Winter OLYMPICS

New ZeAland AT THE 2006 Winter OlympiCS

The concepts seem quite relevant, discussing Estonia and various Olympics-related themes. Now let us examine the query's top-10 concepts.

ESTONIA

ECONOMY OF ESTONIA

ESTONIA AT THE 2000 SUMMER OLYMPICS

ESTONIA AT THE 2004 SUMMER OLYMPICS

ESTONIA NATIONAL FOOTBALL TEAM

ESTONIA AT THE 2006 WINTER OLYMPICS

BALTIC SEA

EUROZONE

TIIT VÄHI

MilitaRY OF ESTONIA

Technically, this document was correctly retrieved by the system, with three of the top concepts shared between query and document. But why were these sports-related concepts generated for this query, despite the query's bearing no relation whatsoever to sports?

The Wikipedia articles from which these sports-related concepts were derived contain no mention of the word "economy," but do contain many instances of the word "Estonia." Thus, the tf.idf score used to compute the weight for the word "Estonia" 
in these sports-related concepts was very high. Hence, even when the query contains other words (such as "economy") for which the weight of these sports-related concepts is very low, the ESA vector for the entire query still assigns them a high weight. As a result, Estonian sports-related documents are ranked too high and are incorrectly retrieved by the system, degrading overall performance. The query concept vector does include concepts related to Estonia's economy, such as ECONOMY OF ESTONIA, TIIT VÄHI (Estonia's prime minister during the country's major economic transformation period) and EUROZONE, but these are not effective in removing the nonrelevant sports results. In this respect, the effect is similar to that of query drift [Mitra et al. 1998] that is caused by excessive text-based query expansion.

Our observation, then, is that since the ESA classifier is created from a noisy unstructured information source, and one that is different from the target corpus, the initial concept vector might carry noise and ambiguities. To counter such problems, we hypothesized that the concept vector should first be tuned to better fit the corpus it is querying. This is similar to the idea that a corpus-based similarity thesaurus [Qiu and Frei 1993] is better than a general-purpose one.

An ESA vector has two candidates for such tuning: the subset of concepts and the weights assigned to them. To check whether tuning should be performed for both of them, we ran the same tests as before, but with all query concept weights set to a uniform value. We found that this change hardly made any difference in performance, and this conclusion was also verified in similar tests in later experiments. Thus, we conclude that tuning the original concepts is useful only when altering the set of concepts to be used. We will focus on this in the next section.

\section{SELECTIVE ESA-BASED RETRIEVAL}

We have shown that the basic ESA concept-based representation of a query or a document may be ambiguous and noisy, requiring tuning before it can be used efficiently. Before we propose tuning methods, we must decide where in the retrieval process the tuning should be applied. As the concept-based representation is used in both the document indexing and query processing stages, it would seem reasonable to suggest that tuning should also be done in both.

We chose, however, to focus on the query processing stage only. The main reason was that queries are much shorter than documents or even passages. For a longer text fragment, the generated concepts reinforce the main topics in the text and noise is restricted, whereas fragments such as short queries (typically 2-3 words in TREC Adhoc datasets) generate concepts that still contain much noise. In addition, tuning a document's representation during the indexing phase is problematic because it lacks the context provided by a given query, and a certain feature may be considered noise for one query but informative for another. Finally, changes in indexing parameters require reindexing, incurring extensive experimentation costs.

\subsection{Feature Selection Using Pseudo-Relevance Feedback}

When ESA was applied to the text categorization task [Gabrilovich and Markovitch 2006], it was vulnerable to the same problems we have just described. Nevertheless, the researchers overcame these problems by employing aggressive Feature Selection (FS). FS methods use labeled training examples to evaluate the utility of candidate features [Guyon and Elisseeff 2003]. In text categorization, these examples are provided as part of the task data. In contrast, the IR task inherently lacks any labeled training data; hence applying FS to information retrieval will require finding an alternative method of evaluating the utility of features (concepts in our case). 
For this purpose, we consider a feature of IR systems called relevance feedback [Rocchio 1971], where the user provides relevance judgments on an initial set of retrieved results. This feedback is then used to reformulate the query and retrieve an improved set of results, thus it can be considered a type of labeled training data for IR. Relevance feedback can also be automated to alleviate the need for users' involvement, by assuming that the top ranked results (documents or passages) in the initial retrieved set are relevant [Salton and Buckley 1990]. This method is commonly referred to as Pseudo-Relevance Feedback (PRF).

Inspired by PRF, we decided to use the results of keyword-based retrieval as a source for evaluation in our FS process. Our updated retrieval method will thus become twophased, first performing keyword-based retrieval, then using its results to tune the query concepts and perform concept-based retrieval.

Next, we had to decide which subsets of the results are to be used. Most of the work on PRF used the top ranked documents or passages [Ruthven and Lalmas 2003; $\mathrm{Xu}$ and Croft 2000] as pseudo-relevant documents (or positive examples). Some researchers chose to include also pseudo-nonrelevant documents (or negative examples), by using the bottom-ranked documents [Huang et al. 2006; Yan et al. 2003], while others found no improvement in doing so [Buckley and Robertson 2008; Kaptein et al. 2008]. We chose to use both positive and negative examples, as the initial query representation includes irrelevant concepts to be removed (for which we believe negative examples will be useful), in addition to missing relevant concepts (for which the positive examples alone are sufficient).

One may argue that, for the purpose of negative examples, randomly selected documents may make a better choice, in particular for queries with many relevant documents. [Singhal et al. 1997] analyzed a similar claim, when suggesting which documents should be used as nonrelevant ones for learning a query profile for information filtering. They showed that sampling nonrelevant documents from the "query zone" (meaning the set of nonrelevant documents that are similar enough to the query) is better than sampling from the entire corpus (minus the relevant documents) when it comes to choosing features that are strong indicators of relevance.

Like the findings of [Singhal et al. 1997], our early findings showed that using the bottom-ranking documents (a "query zone" equivalent) as nonrelevant examples produced better results than using random documents. We also found early in our experimentation that keyword-based passages significantly outperformed full documents. This can be explained by the more coherent concepts produced by concise passages, similar to our findings in Section 3.3.2.

Following these findings, our algorithm will be using the top ranking keyword-based passages as positive examples, and the bottom ranking passages as negative examples. The next section will describe an algorithm for ESA-based retrieval that uses these pseudo-relevant examples to tune and select the query features.

\subsection{Selective ESA-Based Retrieval Algorithm}

Now that we have decided on a framework for evaluating features, let us describe the integration of FS into the general ESA-based retrieval algorithm. Since we chose to perform FS only on the query representation, the indexing algorithm is unchanged and remains as described in Figure 2, and we shall now elaborate on the revised retrieval algorithm provided in Figure 5.

First, as in the nonselective algorithm, the textual query $\vec{q}$ is represented by an ESA concept vector $\vec{F}_{q}$. Then, the first $n$ results ranked by keyword-based retrieval for $\vec{q}$ are fetched. The top $k$ of these $(k \ll n)$ are tagged as pseudo-relevant, or positive examples, and the bottom $k$ are tagged as pseudo-nonrelevant, or negative examples. 


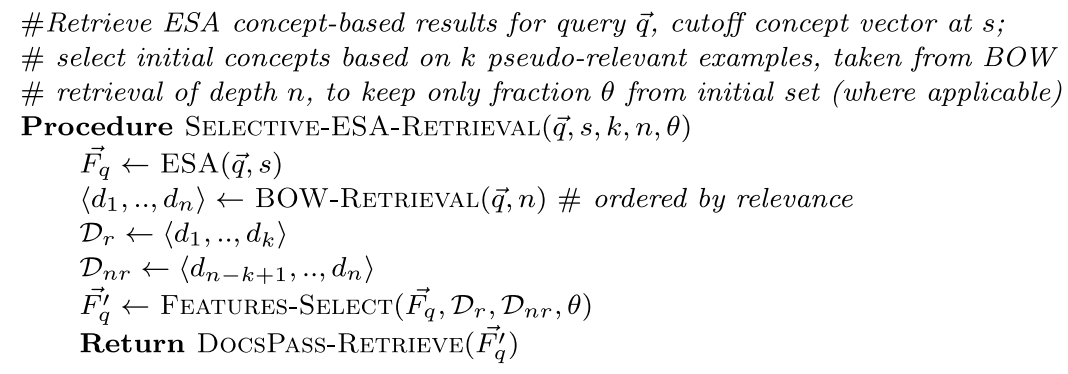

Fig. 5. Selective ESA-based retrieval.

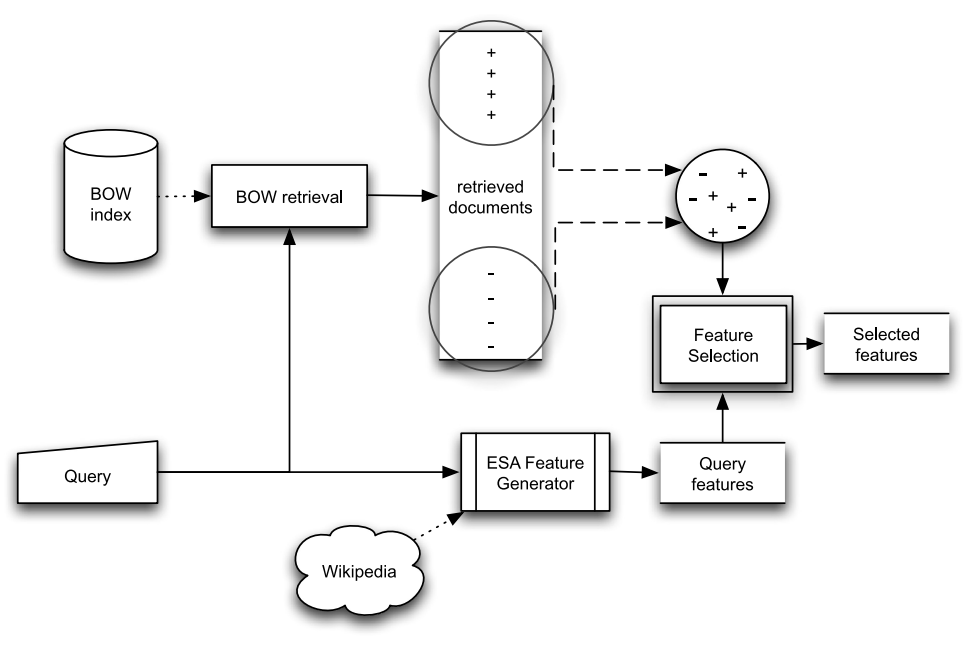

Fig. 6. The PRF-based feature selection process.

Feature selection is then applied to these examples in order to select the best performing concepts in $\vec{F}_{q}$, resulting in a modified ESA vector $\vec{F}_{q}^{\prime}$. Note that in practice, FS manipulates a sparse representation of the vector, hence set operations that are used in the algorithms are with respect to such representation. Finally, concept-based retrieval is performed using $\vec{F}_{q}^{\prime}$ and results are returned. The entire process is illustrated in Figure 6.

Given this generic algorithm and information on positive and negative examples, several actual FS methods can be suggested to implement the generic FEATURESSELECT() step in the algorithm. In the following subsections we propose and experiment with three such FS methods.

4.2.1. Feature Selection Using Information Gain. The first FS method uses each feature's individual utility to select a subset of the initial concept-based representation. This utility is measured by the information gained in separating the set of positive and negative examples [Quinlan 1986]. Information Gain (IG) was originally suggested in the context of a decision tree induction method for choosing which feature to branch on, but is also used extensively in feature selection [Yang and Pedersen 1997]. For a feature $f$ and a set $\mathcal{S}$ composed of positive and negative examples, the IG of $f$ is 

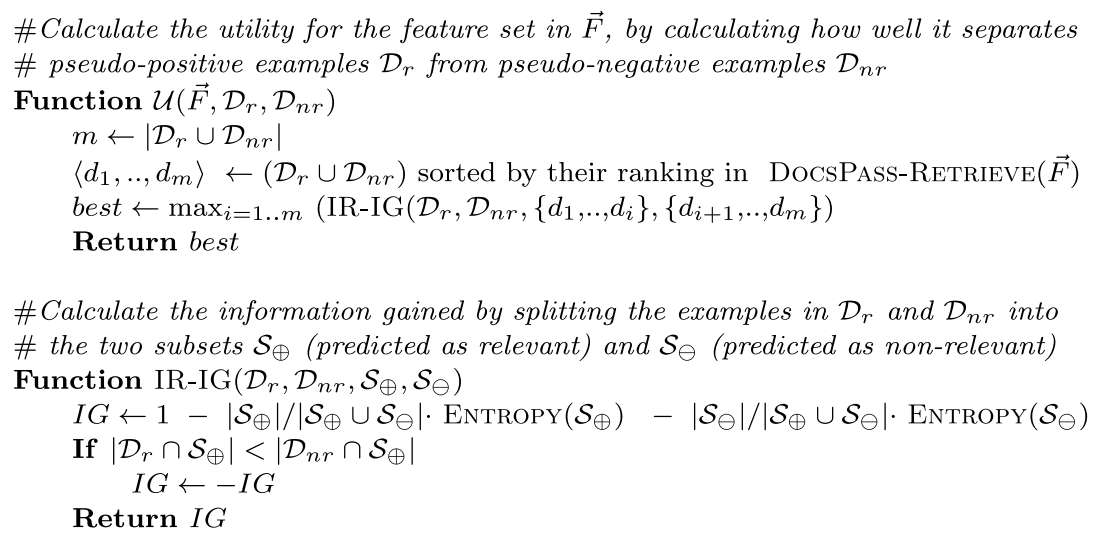

Fig. 7. Utility calculation for a set of concepts to be used in IR.

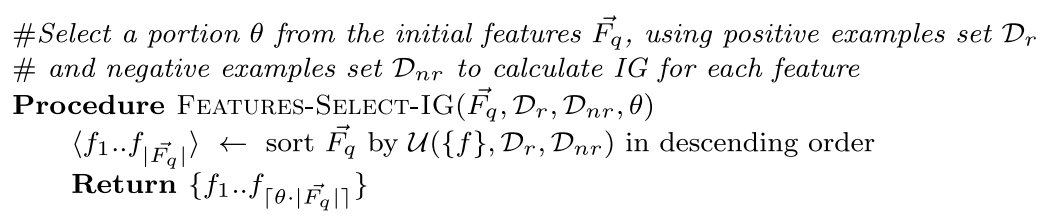

Fig. 8. Selective ESA-based retrieval - IG selection method.

calculated as the change in information entropy $E$ when splitting $\mathcal{S}$ into subsets $\mathcal{S}_{i}$ according to their value of $f$. We have

$$
I G(f, \mathcal{S})=E(\mathcal{S})-\sum_{i} E\left(\mathcal{S}_{i}\right) \cdot \frac{\left|\mathcal{S}_{i}\right|}{|\mathcal{S}|},
$$

where $E(\mathcal{S})$ stands for the information entropy in a set $\mathcal{S}$. In our case, $f$ is an ESA concept, and we define the value of $f$ in each example to be the IR score of that example when $f$ is used as the query. Since such feature values are continuous, they must be discretized in order to split them into subsets and calculate IG. Following [Quinlan 1986], the feature values are discretized by calculating IG for every possible cutoff value, and using the best value as this feature's IG. The complete utility calculation is described in function $\mathcal{U}()$ in Figure 7. The function is generalized to calculate utility for a set of features as well, as some of our FS methods require.

We note that a feature that retrieves primarily negative examples is less useful for IR purposes. The scarcity of relevant documents and the random nature of nonrelevant documents usually imply that very little information is expected to be added by such features. Our version of IG, shown as function IR-IG in Figure 7 and used by the utility function $\mathcal{U}()$, takes that into account by negating the result value when more negative examples are retrieved than positive ones. Negating (rather than setting to zero) also proves useful in producing a value that is easy to sort by, in case we have to select the "least-worst" features. One may argue that features with a large negative value may better be used in a negation retrieval clause (NOT operator), but our experiments showed no added value in doing so, which is probably explained by the incidental and anecdotal nature of those features.

The resulting IG feature selection method is shown in Figure 8. The procedure returns the best performing query features as measured by their IG values, cutting off at the requested level $(\theta)$. 


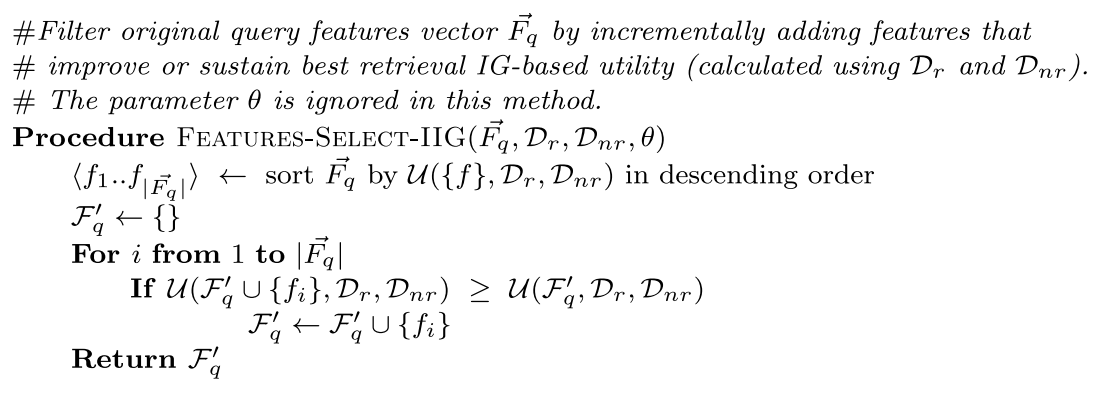

Fig. 9. Selective ESA-based retrieval - IIG selection method using forward selection.

4.2.2. Feature Selection Using Incremental Information Gain. In the previous section, we described a selection method based on the IG value of each individual feature. In our case, however, these features are ultimately used as part of a complete set of query concepts, and dependency between the different features may imply that individual utility calculation is inaccurate. The Incremental Information Gain (IIG) method hypothesizes that feature utility would be better evaluated in the context of a full set of query features. Since examining all subsets of the initial feature set is exponential in the number of initial features and not computationally feasible, we perform a heuristic search in this space using our utility function $\mathcal{U}$ as the heuristic function.

The IIG method builds the representation incrementally, using forward selection or backward elimination [John et al. 1994]. Features are first sorted by their individual IG value, and the candidate query set is an empty one (or the full one, for backward elimination). Then, in each iteration a feature is added to the candidate set (or removed, for backward elimination) if this step does not degrade ${ }^{6}$ current pseudorelevance-based performance, or discarded otherwise. When all features have been evaluated, the algorithm terminates and returns the selected features. In addition to the advantage of evaluating the feature in the context of other features, this method also has the advantage of not requiring a predefined selection level, thus removing one parameter from the system.

Figure 9 shows the IIG selection method when using forward selection. For backward elimination, the algorithm will begin with the full feature set, and in each iteration attempt to eliminate the lowest-performing feature, choosing to keep it if its removal harms performance.

4.2.3. Feature Selection Using a Rocchio Vector. In the two previously described FS methods, the set of candidate features were those generated for the query by the ESA feature generator, $\vec{F}_{q}=E S A(\vec{q}, s)$. However, the extremely short queries (1-3 words in the datasets we used) may not suffice to generate and assign high weight to important concepts.

Consider query 415 in TREC-8, “drugs, Golden Triangle." This query refers to an area in southeast Asia that is known for illicit opium production, but since no such single explicit concept existed in our ESA model, the query's top concepts were related to other "golden triangle" meanings, and relevant topic-related concepts were not considered. Employing FS on the generated concepts was naturally not helpful, as the initial candidate set's coverage was not sufficient.

Yet, our ESA model does include other features that could represent the correct "golden triangle" using other concepts, such as ILLEGAL DRUG TRADE, OPIUM,

${ }^{6}$ This condition implies that for forward selection we will keep redundant features, whereas for backward elimination we will remove them. We elaborate on this in the results section. 


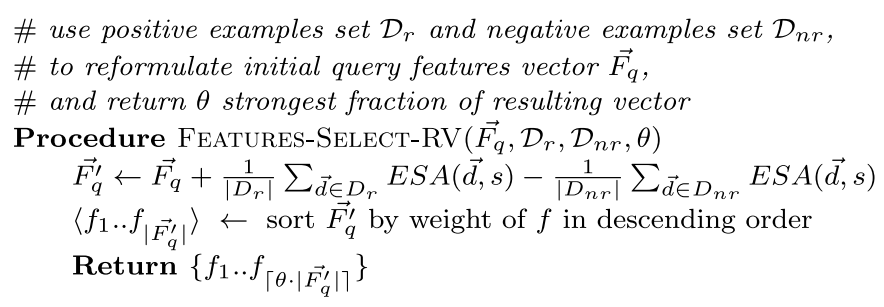

Fig. 10. Selective ESA-based retrieval - RV selection method.

MYANMAR and LAOS (two countries located in this triangle). Such ESA concepts could be generated from texts discussing the correct query interpretation. Since the top retrieved documents for the keyword-based query are expected to be such texts, we may use them to try and compensate for the inaccurate query concepts. Hence, we would like to generate and use these concepts as additional concepts in the set of candidate features to be selected.

We thus propose a new FS method where the augmented set of candidate features is $\vec{F}_{q}=E S A(\vec{q}, s) \cup\left\{E S A(\vec{d}, s) \mid \vec{d} \in D_{r}\right\}$. Now we need to evaluate and select features from this set. Using IG to evaluate how well each feature separates top-ranking documents from bottom-ranking ones is not sound, as the additional features were already taken from the top-ranking documents. Instead, we will use the weights of each feature in each document in the sets of positive and negative examples, average these values into a combined weight, and use the results to select features.

We calculate the features' weights based on Rocchio's algorithm for relevance feedback [Rocchio 1971]. Each feature receives a weight that is the sum of its weights in the original query and in the positive example documents, and then its weights in the negative example documents are subtracted. Finally, the strongest features are kept and the rest discarded. The pseudocode for applying the RV method is provided in Figure 10.

\subsection{Empirical Evaluation}

This section describes experiments carried out using selective ESA-based retrieval with each of the selection methods, and a comparative analysis of the results.

4.3.1. Methodology. We continue using the experimental framework described in Section 3.3.1, and evaluate each suggested selection method with various system parameter settings. The following parameters have been fixed to a predefined value in all these experiments: $s$, the concept vector cutoff, has been set to 50 ; and $n$, the BOW retrieval depth for pseudo-relevance, has been set to the first 1000 results. The system parameters we will be experimenting with are $k$, the pseudo-relevant result set size, and $\theta$, the feature selection aggressiveness level (where applicable).

To further assess the value of feature selection in itself, we also experimented with a fourth, random method, which randomly selects a subset of features of the required size (as defined by $\theta$ ) from the original representation, regardless of the provided examples. We used this method to reject the hypothesis that an observed improvement in performance may solely or partly be attributed to the use of a smaller subset of the original features rather than the specific features selected.

4.3.2. IG Method Results. The IG method has two primary parameters: the number of pseudo-relevant examples $(k)$ and the selection level $(\theta)$. Figure 11 shows retrieval performance (averaged over all queries in each dataset) as a function of $\theta$ for several values of $k$, compared with a baseline that performs no FS at all. Both datasets show 

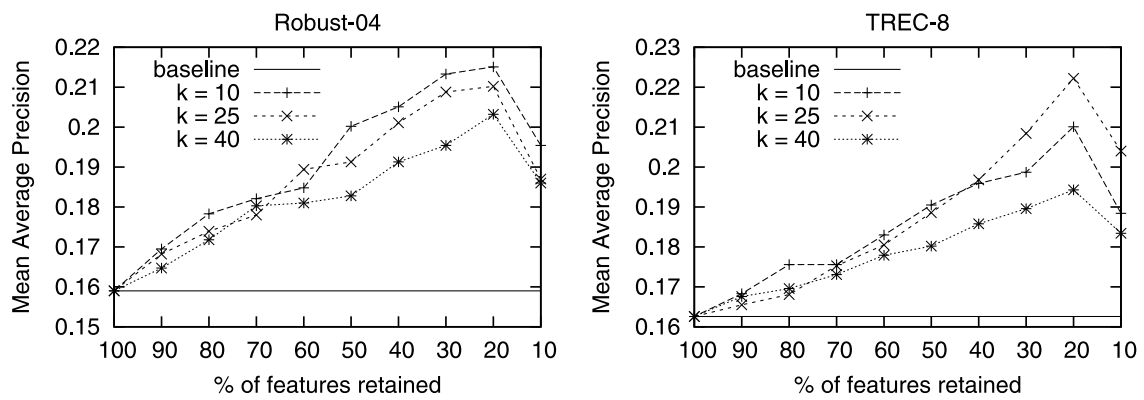

Fig. 11. Concept-based performance as a function of a fraction of the concepts selected $(\theta)$, IG method.
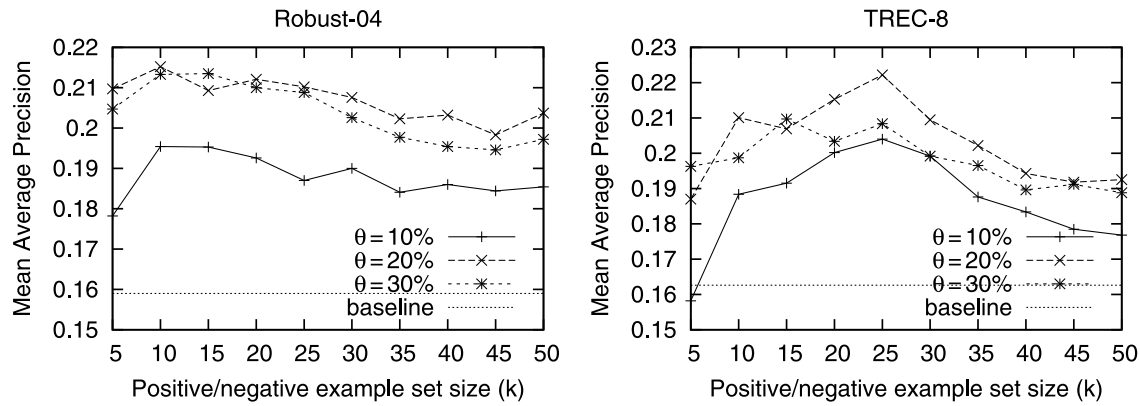

Fig. 12. Concept-based performance as a function of the number of pseudo-relevant examples (k), IG method.

similar behavior, with FS performance consistently improving as selection level increases, peaking at $\theta=20 \%$ (which implies retaining 10 out of the initial 50 features). More aggressive selection is already damaging, probably as the result of removing useful features along with nonrelevant ones.

Figure 12 shows the same experiment from a different perspective, with performance as a function of $k$ for several values of $\theta$. The number of examples used seems to influence performance less than selection level, except when too few examples are used $(k=5)$, resulting in insufficient information for IG to be reliable. Nevertheless, adding more and more examples degrades rather than improves performance. This may be attributed to the decrease in actual relevance of the pseudo-relevant examples when taken from lower-rank positions.

4.3.3. IIG Method Results. The IIG method requires only one parameter to be set, the size of the positive/negative example set $(k)$. In addition, the algorithm may be run in forward selection or in backward elimination mode. Figure 13 shows retrieval results for different values of $k$ in both modes, compared with results of the initial baseline query.

In both datasets, the IIG method shows consistent improvement over the performance of the baseline. The results also show the forward selection approach consistently outperforming the backward elimination approach. One reason we found for this was the inherent filtering of redundant features in backward elimination. If a certain query has two highly informative but similar features, it is quite possible that each alone will be sufficient to perfectly separate the positive from negative examples. Then, backward elimination will eliminate one of them, as its removal does not degrade performance, although in a full corpus retrieval that additional feature could have contributed to the query's performance. This may also explain why the 

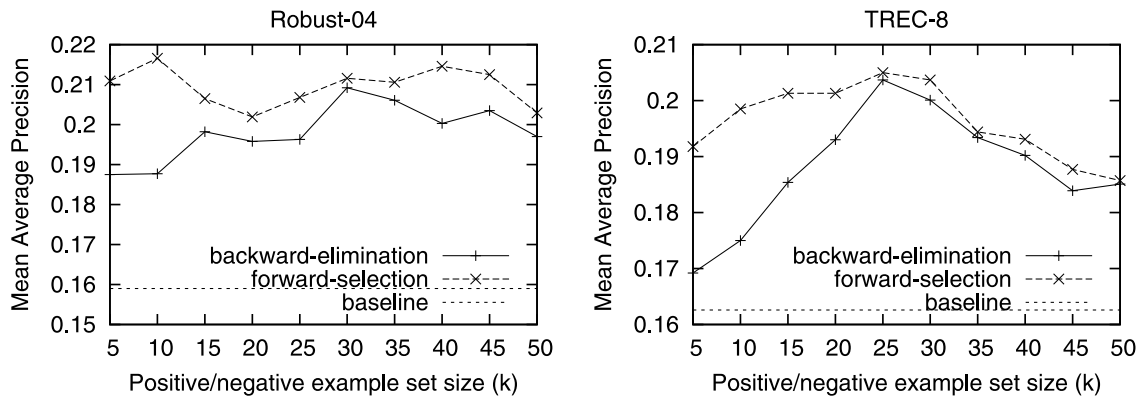

Fig. 13. Concept-based performance as a function of the number of pseudo-relevant examples (k), IIG method.
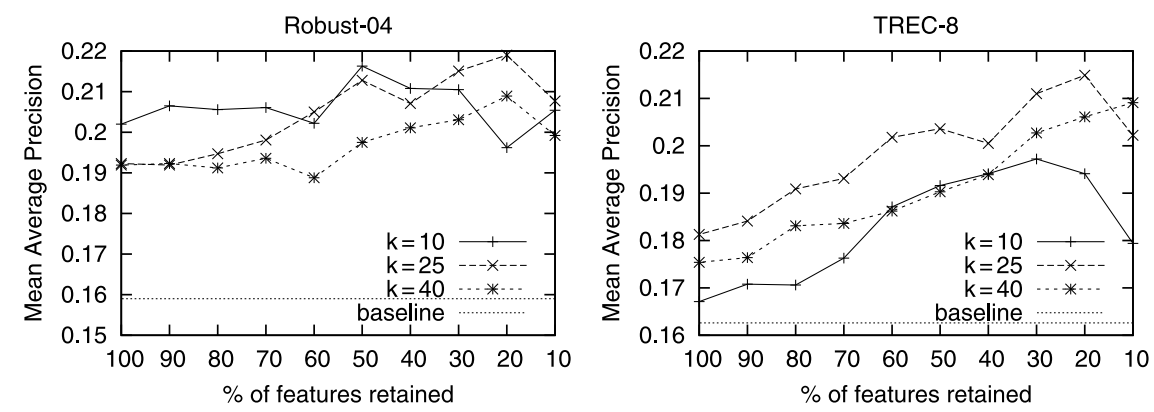

Fig. 14. Concept-based performance as a function of a fraction of the concepts selected $(\theta)$, RV method.

difference between forward selection and backward elimination is greatest when very few examples are used.

We also experimented with another variation of the IIG method, where the weights of each examined feature were recalculated in each iteration. In this version, the next feature to add will be the one to maximize the local value $\mathcal{U}\left(\mathcal{F}_{q}^{\prime} \cup\{f\}\right)$ rather than the global $\mathcal{U}(\{f\})$ used in the algorithm described in Figure 9. Despite this version being more in line with common practice hill climbing implementations, it performed well below the global one. We suspect this is due to the inaccurate nature of the example documents, which increase the chance for local maxima.

4.3.4. RV Method Results. The RV method, like IG, requires setting two parameters, $k$ and $\theta$. Like the graphs in the previous sections, the graphs in Figures 14 and 15 show the impact of these parameters on the system's performance. But whereas with the IG method the query reverts to the original query at $\theta=100 \%$, this is not the case with the RV method. Even without any selection, the query changes as a result of adding the features generated from the positive example documents and of the reweighting step.

Figure 14 shows that even without selection, performance is better than the baseline, and that the improvement generally increases with the selection level (except for the very high selection levels). Figure 15 shows that using a very small set of examples $(k=5)$ yields poor results, with performance improving and stabilizing as more examples are provided. Once performance stabilizes, adding further examples does not seem to make much difference. The impact of FS is also clearly demonstrated, with the $\theta=100 \%$ curve mostly lower than the highly selective curves. 

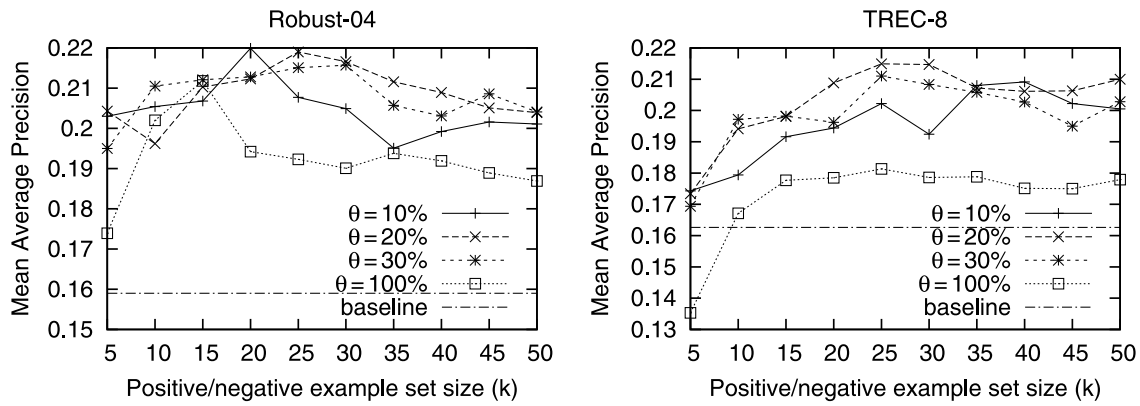

Fig. 15. Concept-based performance as a function of a fraction of the number of pseudo-relevant examples (k), RV method.
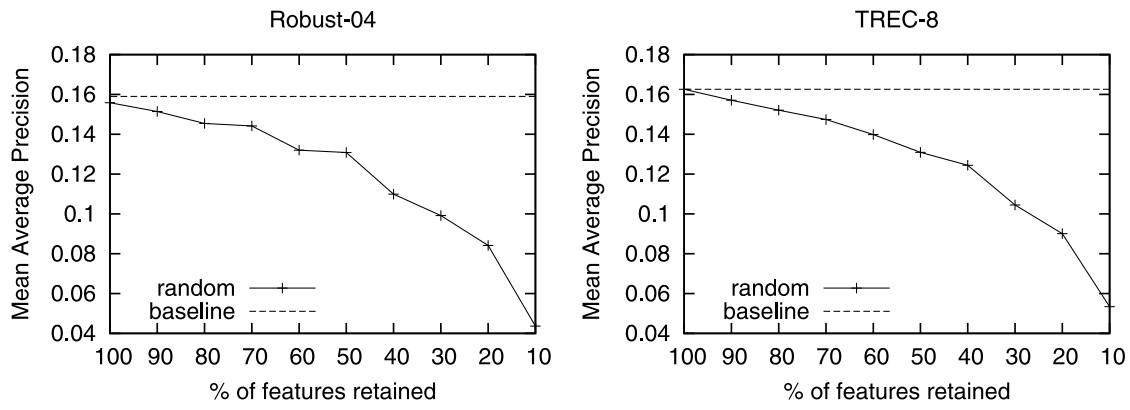

Fig. 16. Performance of random selection method, averaged over 10 runs each

4.3.5. Random Selection Results. We replaced the PRF-based selection process with a random one. A subset of the required size (determined by the parameter $\theta$ ) was randomly sampled from the initial query features set for each query, and retrieval results for these randomized concept-based queries were evaluated. This process was then repeated 10 times (for each choice of $\theta$ ). The parameter $k$ was irrelevant for these experiments, as the examples were not used in any way.

The results in Figure 16 show a continuous decrease in performance as more features are randomly removed from the initial set. This clearly indicates that the improvement shown by previous methods must be attributed to the specific set of features chosen, rather than just the act of using a smaller set of features.

4.3.6. Parameter Tuning through Training. All selection methods shown in this section rely on one or two system parameters, whose values may have a significant impact on system performance. These parameters can be tuned if a set of queries is provided with relevance judgments on result documents. We used a third dataset, TREC-7 [Voorhees and Harman 1998], which shares the same corpus as TREC-8 and Robust-04 but has a different set of queries, to perform parameter tuning.

We operated the system on TREC-7 queries with the three proposed FS methods and varied the parameter value ranges. The resulting best performance values obtained were: for IG FS $\langle k=10, \theta=30 \%\rangle$, for IIG FS $\langle$ forward selection, $k=10\rangle$, and for RV FS $\langle k=35, \theta=20 \%\rangle$. All of these values fall well within the top performing value ranges (though not always the peak values) in TREC-8 and Robust-04. This result, coupled with the similarity of the system's performance graphs over the TREC-8 and Robust04 datasets, suggests that relatively consistent system behavior can be expected, 
and ESA-based systems may be tuned on one set of queries and then used on other test sets.

\subsection{Analysis}

Having evaluated each of the suggested FS methods, let us examine the results in greater depth. We have demonstrated that feature selection on the query concept vector is effective in obtaining better retrieval results, and that this improvement is not the result of merely using a smaller set of concepts. Now let us compare the effectiveness of each method, in order to draw some general conclusions as to what scenario they may be best suited for.

The IG method exhibits good peak behavior, but it seems to be highly sensitive to the chosen selection level $\theta$. Tuning the system parameters using training data, if available, may significantly alleviate this problem, as shown by the tuning experiment we conducted.

The IIG forward selection method appears to perform better than backward elimination. This method requires tuning only a single parameter: the number of examples to be used. It would therefore be the preferred choice when no training data is available. Its performance, though, is slightly lower than IG, and it is still quite sensitive to the $k$ parameter value.

The RV method performs slightly worse than IG for small example set sizes, probably due to its overdependence on the quality of these examples (as they are a source of generating features, not just filtering harmful ones). For larger example sets (in our case, $k>15$ ), it performs comparably to the IG method. In addition, the RV method appears more robust than the other two, in that it yields overall good results for a broader range of parameter settings, rather than a pinpointed peak, and therefore will depend less on accurate parameter tuning.

Let us now revisit the Estonian economy example from Section 3.3.3. The revised query, after being processed by the RV method (as an example), is as follows.

ECONOMY OF ESTONIA

MONETARY POLICY

ESTONIA

EURO

ECONOMY OF EUROPE

NEOLIBERALISM

TIIT VÄHI

Prime Minister of Estonia

EUROZONE

NORDIC COUNTRIES

The noisy sports-related concepts that appeared in the initial features are now filtered out of the query, as they appear very rarely (if at all) in the concepts of both sets of positive and negative examples. Other concepts that may seem relevant at first, such as ESTONIA and BALTIC SEA, are filtered out for being too broad, appearing frequently in the concepts of both example sets. Concepts that are highly relevant to Estonia's economy, such as ECONOMY OF ESTONIA, TIIT VÄHI, and EUROzONE, are retained in the top positions, while other relevant ones percolate upwards. In addition, the RV method also added NEOLIBERALISM, an economy-related concept relevant to Estonia's economy that was not included in the original query concepts but appeared frequently in the concepts of positive examples.

To summarize, Figure 17 shows the improvement over the baseline for the three methods for $\theta=20 \%$. On the basis of this figure, we can state that adding FS to ESA 

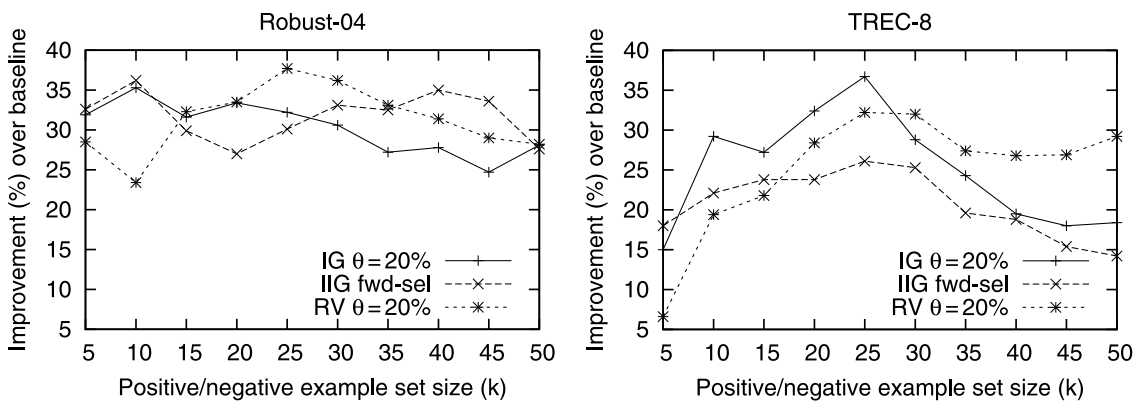

Fig. 17. Best performing runs for all three FS methods.

concept-based retrieval can significantly improve retrieval results, with improvement of up to $40 \%$ over the nonselective ESA baseline in both datasets.

Note that even with this significant improvement, retrieval performance still stands at just over $85 \%$ of our BOW baseline. We believe that an inherent bias in the evaluation methodology may contribute to this low measured performance, and we will elaborate on this issue in Section 5.3.5. However, these results are sufficiently improved that we can now proceed with our plan to augment the BOW representation with the selected ESA features for our final concept-based retrieval model.

\section{FUSED SELECTIVE ESA-BASED RETRIEVAL}

A large body of research [Croft 2000; Fox and Shaw 1994; Lee 1995; Vogt and Cottrell 1999] shows that combining (also known as "fusion" of) retrieval methods may improve final results. Fusion of ranking approaches is known to achieve best results when the methods to be combined are substantially different in their approach [Lee 1995]. With the significant difference between BOW and ESA representations, we expect that combining them will also yield better results. This idea is further reinforced by the findings of [Gabrilovich and Markovitch 2005] in applying ESA to text categorization, which showed that augmenting the BOW representation with ESA concepts outperforms each individual representation alone.

\subsection{Fused Selective ESA-Based Retrieval Algorithm}

A survey of combining approaches can be found in [Croft 2000]. In our study we use the simple, widely used model of linear combination [Vogt and Cottrell 1999], where document scores are weighted sums of the scores assigned by the individual retrieval methods to be fused, with weighting determined using training data. Before summing, document scores are normalized to account for the different ranges in score values, as suggested by [Lee 1995].

Once both retrieval results (concept based and keyword based) are normalized, document scores are then weighted and summed using the weight factor $w$, provided as an additional parameter. The pseudocode for this algorithm is described in Figure 18. The value for this parameter can be obtained using parameter tuning on a dataset with relevance judgments.

\subsection{The MORAG System}

Let us now recap the entire resulting system, which we named MORAG, ${ }^{7}$ as illustrated in Figure 19. First, an ESA model is built from Wikipedia or another source, as

\footnotetext{
${ }^{7}$ Morag is the Hebrew word for flail, an agricultural tool used to separate grain from chaff.
} 


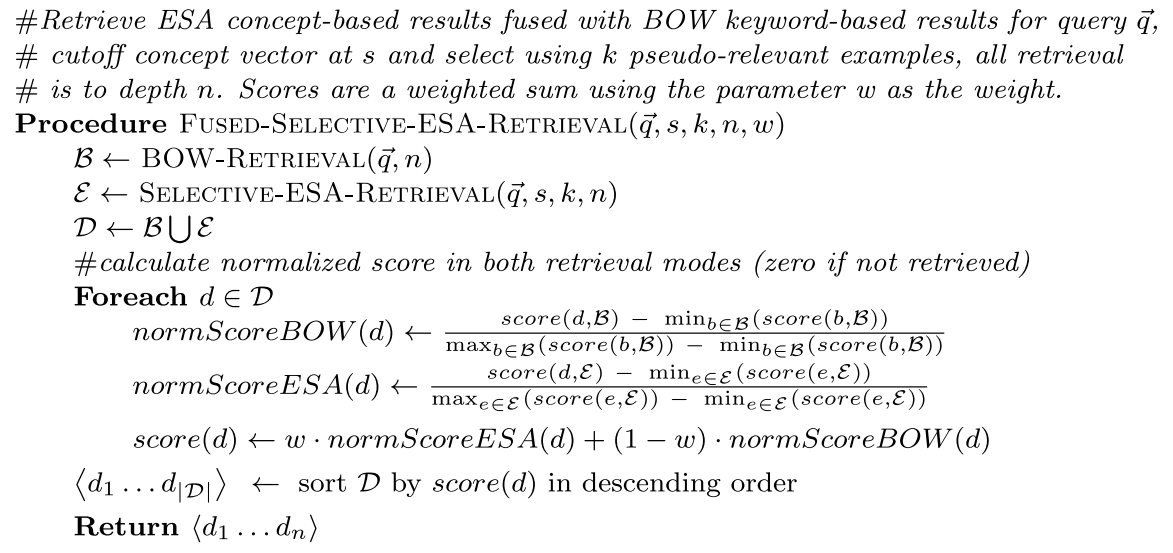

Fig. 18. Fused selective ESA-based retrieval - the MORAG algorithm.

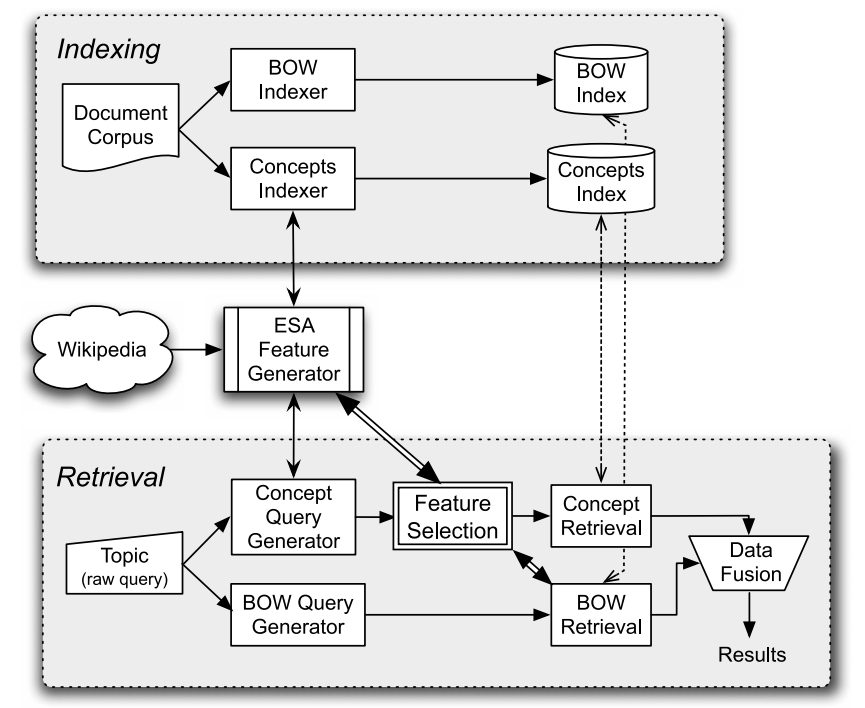

Fig. 19. The MoraG solution architecture.

described in [Gabrilovich and Markovitch 2006]. During the indexing stage, MoRAG indexes the corpus in both BOW and ESA representations. Then, at retrieval time, the BOW query is submitted; its results are kept for the fusion phase and also fed into the FS module, together with the ESA query representation. After FS is complete, the resulting features are used to perform a concept-based retrieval, and the results of the concept-based and keyword-based retrieval runs are fused to produce the final MORAG results.

Note that in our implementation of MORAG we have used the same BOW subsystem for both purposes: generating pseudo-relevant examples, and fusion to concept-based results. However, other implementations using different BOW retrieval systems for each of these purposes are also possible. 
Table I. Performance of MoRAG Using Tuned Parameter Values and Optimal Parameter Values

\begin{tabular}{cc|cccc}
\hline Dataset & Baseline & $\begin{array}{c}\text { MorAG - IG } \\
\text { tuned }\end{array}$ & $\begin{array}{c}\text { MorAG - IIG } \\
\text { tuned }\end{array}$ & $\begin{array}{c}\text { MorAG - RV } \\
\text { tuned }\end{array}$ & $\begin{array}{c}\text { MoRAG } \\
\text { optimal }\end{array}$ \\
\hline TREC-8 & 0.2481 & $0.2864(+15.4 \%)$ & $0.2734(+10.2 \%)$ & $0.2888(+16.4 \%)$ & $0.2947(+18.8 \%)$ \\
Robust-04 & 0.2622 & $0.2914(+11.1 \%)$ & $0.2923(+11.5 \%)$ & $0.2879(+9.8 \%)$ & $0.3010(+14.8 \%)$ \\
\hline
\end{tabular}

Improvement percentage over baseline is shown in parentheses next to each result.
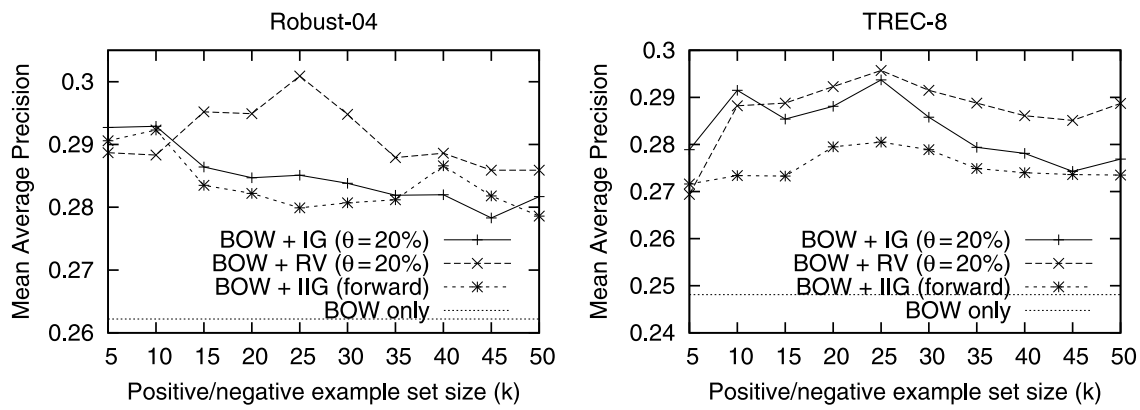

Fig. 20. MORAG performance as a function of a fraction of the number of pseudo-relevant examples (k), all methods.

\subsection{Empirical Evaluation}

We ran a set of experiments to evaluate the performance of the MORAG system and to analyze its robustness and further potential.

In addition, we evaluated MORAG in combination with and in comparison to top performing systems in TREC-8. As [Armstrong et al. 2009] recently pointed out, it is not sufficient for IR researchers to show improvement over their own baseline, rather they should strive to show that their method can improve over systems that are already highly effective. We will show that our method is indeed capable of doing that.

5.3.1. Methodology. The experimental methodology generally follows that of the previous section. Specifically, in this algorithm, we also need to tune the value for the parameter $w$. We used the TREC-7 dataset for this purpose too, selecting the parameter value that maximized the performance of MORAG on TREC-7, which was found to be $w=0.5$ for the combination of ESA and Xapian BOW.

5.3.2. Morag Results. Table I shows results for both TREC- 8 and Robust-04 datasets for all three FS methods, with parameters tuned on the TREC-7 dataset. The last column shows the system's performance with optimal choice of parameters, as an indicator of what further improvement can be achieved by better parameter tuning.

The results show an impressive improvement over the BOW baseline for all FS methods. Parameter tuning yields reasonable results: $55 \%-85 \%$ of the optimal performance. We checked the statistical significance of the results using a paired two-tailed t-test, and all the results were significant at $p>0.95$.

Figure 20 compares performance for the different selection methods in MORAG, for various values of the parameter $k$, assuming that the parameter $\theta$ is easier to optimize due to its peak behavior (or its irrelevance for IIG). The results show the RV method achieves best results for sufficiently large values of $k$.

Figure 21 shows results for one specific choice of selection method and level, comparing the performance of the fused system with that of its components. The graph demonstrates how fusion with ESA-based results improves the system's performance by an increment that is correlated with the ESA system's performance, as expected. 

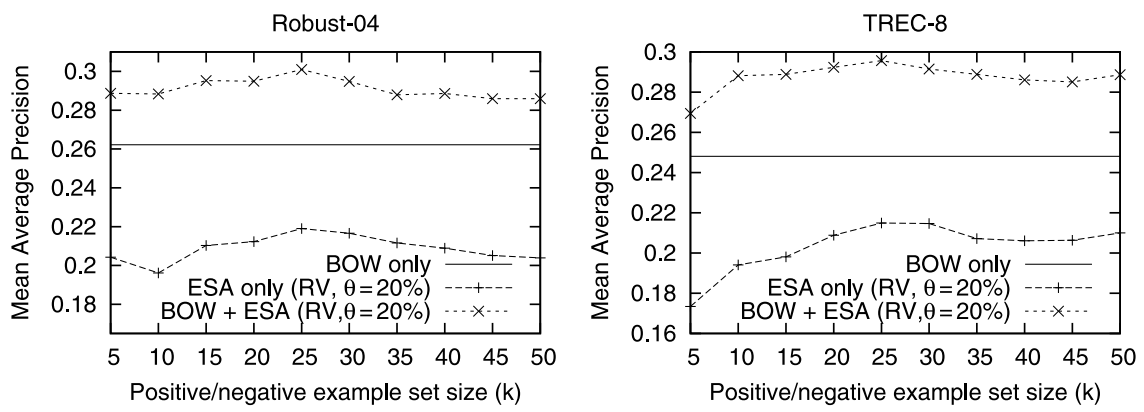

Fig. 21. Comparison of fused results with results of each fused subsystem on its own (for a single choice of FS method and selection level).

Note that despite the relatively low performance of the ESA run, fusion still yields good improvement. Similar behavior is also observed for the other methods and selection level values.

5.3.3. Fusion with Alternative BOW Subsystems. The previous experiments were carried out using our choice of an experimental BOW system (Xapian). However, since MORAG is modular, it can be used with any other BOW component, and we were interested in assessing the system's robustness over different (and better performing) BOW systems.

We used two additional effective and common retrieval approaches implemented in the Lemur toolkit ${ }^{8}$ : a TF.IDF-weighted vector space model with pseudo-relevance feedback (we denote this run FB-TFIDF), and a language model based on KL-divergence using Dirichlet prior smoothing (denoted LM-KL-DIR). We used the "out of the box" Lemur implementations with default parameter values, and set the MORAG-specific parameters for these systems by parameter tuning on TREC-7.

In addition, we wanted to use systems that achieved the highest results in the original TREC runs. Normally, such experimentation is not feasible, since these systems (or their exact detailed implementation) are usually not available, and evaluations of this kind are not common in IR. However, since MORAG performs fusion on the result set level (rather than change the core ranking functions), such comparisons are possible in our case using only the target systems' output. TREC provides access to past participants' raw results, and we used this data as additional BOW systems.

When determining which TREC systems are best to compare with, we searched for those that employed standard BOW approaches, were among the top performing on the evaluated datasets, and that participated in TREC-7 (with no major internal changes) so that we could also perform parameter tuning using their TREC-7 results. We could not find candidates in the Robust-04 dataset that were good enough for this comparison; hence, we will show results only on the TREC-8 dataset.

Note that the BOW system is used twice in MORAG: once as a source for PRF, and once for fusing the results. However, since relevance feedback in MORAG is passagebased, and the system outputs we had access to were document-based results, we still had to use our own BOW baseline for the PRF stage.

We used BOW results from the Okapi [Robertson and Walker 1999], PIRCS [Kwok et al. 1999], and AT\&T [Singhal et al. 1999] teams, which were three of the top performing systems of TREC-8 participants using short queries. The Okapi and AT\&T teams augmented standard BOW retrieval with extensive query expansion methods

${ }^{8} \mathrm{http}: / / w w w . l e m u r p r o j e c t . o r g /$ 


\begin{tabular}{|c|c|c|c|}
\hline $\begin{array}{c}\text { BOW } \\
\text { system }\end{array}$ & Baseline & $\begin{array}{l}\text { MORAG } \\
\text { (tuned) }\end{array}$ & $\begin{array}{c}\text { MoRAG } \\
\text { (optimal) }\end{array}$ \\
\hline Xapian & 0.2481 & $0.2864(15.4 \%)$ & $0.2947(18.8 \%)$ \\
\hline LM-KL-DIR & 0.2498 & $0.2877(15.2 \%)$ & $0.2924(17.1 \%)$ \\
\hline FB-TFIDF & 0.2697 & $0.2829(4.9 \%)$ & $0.2951(9.4 \%)$ \\
\hline Okapi & 0.2787 & $0.3042(9.1 \%)$ & $0.3065(10.0 \%)$ \\
\hline $\mathrm{AT} \& \mathrm{~T}$ & 0.2853 & $0.2977(4.3 \%)$ & $0.3096(8.5 \%)$ \\
\hline PIRCS & 0.3063 & $0.3211(4.8 \%)$ & $0.3239(5.7 \%)$ \\
\hline
\end{tabular}

Improvement percentage is provided in parentheses.

Statistically significant results are marked in boldface.

based on PRF, while the PIRCS team used a system that combined different BOW retrieval models (probabilistic and language modeling). As stated earlier, our relevance feedback utilized Xapian passage-based results for all runs, and the ESA FS method used in these experiments was IG. All three teams stated in their publications that their system was virtually the same as that used in TREC-7; hence we take the parameter tuning on TREC-7 to be valid for these systems as well.

Table II shows the improvement gained by using each of these systems as the BOW component in MORAG. The third column shows results when fusing with tuned parameter values as described before, while the fourth column shows results for optimal parameter values. We evaluated these results for statistical significance as well, and significant results are marked in boldface.

These results demonstrate that improvement can also be achieved with top performing BOW systems, although the added value of the fusion was lower in those cases. This is understandable, given the current relatively low performance of ESA retrieval alone, and considering that successful fusion is known to require the fused systems to have comparable performance levels [Croft 2000].

5.3.4. Comparison to Fusion of BOW Systems. Fusing results from two retrieval systems is known to be a potential source of improvement in itself [Croft 2000], regardless of the underlying text representations. To assess the true contribution of ESA concepts to the results shown thus far, we wanted to measure what portion of the improvement gained by MORAG can be attributed solely to the act of fusing results. To do so, we compared the improvement attained by MORAG with that attained by fusing the baseline BOW results with results of another BOW system whose measured performance is similar to that of our concept-based retrieval subsystem.

We compared optimal results for MORAG with optimal- $w$ results of fusion with several other TREC-8 participants who applied the BOW approach and used short queries: RMIT [Fuller et al. 1999], ACSys [Hawking 1999], and INQUERY [Allan et al. 1999]. These three system runs had a comparable or slightly higher MAP score than our ESA-based run, and fusing them with each of the BOW systems in the table provides an indication of the value of fusion itself. We used optimal rather than tuned $w$ values, since only one of these participant groups (INQUERY) stated that no changes were made between TREC-7 and TREC-8, and hence training on TREC-7 was not sound.

Table III shows the results of these experiments. For comparison, the last column lists the optimal MORAG improvements again. The obtained results are much poorer than MORAG's and most are not statistically significant, despite being produced by fusion with systems that perform slightly better than our ESA retrieval method. This 
Table III. Comparison of MORAG TREC-8 Results (optimal parameter values) with TREC-8 Results of BOW-BOW Fusion (optimal $w$ values)

\begin{tabular}{|c|c|c|c|c|}
\hline $\begin{array}{l}\text { BOW } \\
\text { system }\end{array}$ & $\begin{array}{c}+\mathrm{RMIT} \\
(\mathrm{MAP}=0.2236)\end{array}$ & $\begin{array}{c}\text { +ACSys } \\
(\mathrm{MAP}=0.2309)\end{array}$ & $\begin{array}{l}\text { +INQUERY } \\
(\mathrm{MAP}=0.2325)\end{array}$ & $\begin{array}{c}\text { MORAG } \\
(\mathrm{MAP}=0.2223)\end{array}$ \\
\hline Xapian & $0.2524(+1.7 \%)$ & $0.2569(+3.5 \%)$ & $0.2586(+4.2 \%)$ & $0.2947(+18.8 \%)$ \\
\hline Okapi & $0.2921(+4.8 \%)$ & $0.2882(+3.4 \%)$ & $0.2903(+4.1 \%)$ & $0.3065(+10.0 \%)$ \\
\hline $\mathrm{AT} \& \mathrm{~T}$ & $0.2943(+3.2 \%)$ & $0.2933(+2.8 \%)$ & $0.2897(+1.5 \%)$ & $0.3096(+8.5 \%)$ \\
\hline PIRCS & $0.3086(+0.8 \%)$ & $0.3068(+0.1 \%)$ & $0.3075(+0.4 \%)$ & $0.3239(+5.7 \%)$ \\
\hline
\end{tabular}

Statistically significant results are in boldface.

Table IV. Additional IR Evaluation Measures for TREC-8 Results Using Several BOW Baselines

\begin{tabular}{c|ccccccc}
\hline $\begin{array}{c}\text { BOW } \\
\text { system }\end{array}$ & MAP & P@5 & P@10 & $\begin{array}{c}\text { relevant } \\
\text { retrieved }\end{array}$ & $\begin{array}{c}\text { overlap of } \\
\text { relevant }\end{array}$ & $\begin{array}{c}\text { non-rel } \\
\text { retrieved }\end{array}$ & $\begin{array}{c}\text { overlap of } \\
\text { non-rel }\end{array}$ \\
\hline Xapian & 0.2481 & 0.484 & 0.472 & 2735 & & 20106 & \\
w/MORAG & 0.2864 & 0.552 & 0.478 & 3062 & 1824 & 19400 & 7115 \\
& $(+15.4 \%)$ & $(+14.0 \%)$ & $(+1.3 \%)$ & $(+12.0 \%)$ & & $(-3.5 \%)$ & \\
w/inq601 & 0.2586 & 0.484 & 0.462 & 2907 & 2299 & 21436 & 15180 \\
& $(+4.2 \%)$ & $(0.0 \%)$ & $(-2.1 \%)$ & $(+6.3 \%)$ & & $(+6.6 \%)$ & \\
\hline Okapi & 0.2787 & 0.552 & 0.488 & 3013 & & 21271 & \\
w/MORAG & 0.3042 & 0.580 & 0.522 & 3168 & 2161 & 20410 & 8531 \\
& $(+9.1 \%)$ & $(+5.1 \%)$ & $(+7.0 \%)$ & $(+5.1 \%)$ & & $(-4.0 \%)$ & \\
w/RMIT & 0.2921 & 0.536 & 0.474 & 3095 & 2370 & 22878 & 13790 \\
& $(+4.8 \%)$ & $(-2.9 \%)$ & $(-9.2 \%)$ & $(-2.3 \%)$ & & $(+12.1 \%)$ & \\
\hline LM-KL-DIR & 0.2498 & 0.468 & 0.442 & 2857 & & 22048 & \\
w/MORAG & 0.2877 & 0.552 & 0.506 & 3087 & 2042 & 20553 & 7759 \\
& $(+15.2 \%)$ & $(+17.9 \%)$ & $(+14.4 \%)$ & $(+8.1 \%)$ & & $(-6.8 \%)$ & \\
w/FB-TFIDF & 0.2717 & 0.488 & 0.444 & 3124 & 2922 & 22450 & 17012 \\
& $(+8.8 \%)$ & $(+4.3 \%)$ & $(+0.5 \%)$ & $(+9.3 \%)$ & & $(+1.8 \%)$ & \\
\hline BOW systems
\end{tabular}

BOW systems are fused with concept-based retrieval (using MORAG) and with another BOW system for comparison.

indicates that the improvement in the previous section cannot be attributed solely to fusion, and demonstrates the added value in the concept-based retrieval component of Morag. This finding is also in line with [Lee 1995], who posited that combining retrieval approaches works best when the representation and weighting schemes differ significantly.

5.3.5. Additional Measures and Analysis. We have shown in the previous sections that fusion with ESA concept-based retrieval produces better results than fusing with BOW systems. We now try to better understand why this is so.

Table IV shows additional IR measures for several of the tested BOW systems, listing measured values for the baseline run of each system (first line), for the MORAG run using that system (second line), and for a run that fuses with another BOW system (fourth line). In this latter run, for Xapian and Okapi we used the best performing fusion in Table III, while the two Lemur runs were fused with each other.

Examining the "P@5", "P@10", and "relevant retrieved" columns, we observe that the improvement in MAP demonstrated by MORAG is not to be attributed primarily to an improvement mainly in recall or mainly in precision; both measures are substantially improved. To further assess the improvement in recall we have also measured the overlap in relevant documents retrieved between each pair of fused 
systems ("overlap of relevant" column). Little overlap between the systems means that there is more chance that each system contributes new relevant documents to the pool, thus higher chances for higher overall recall. However, the Lemur fusion run (LM-KL-DIR w/FB-TFIDF), where 2922 of the final 3124 relevant documents are shared between the two fused runs and yet the overall recall is higher than MoRAG's and nevertheless the final MAP is lower, demonstrates that other factors need to be examined to get the full picture.

The "non-rel retrieved" column measures the number of documents retrieved by each system that were judged by TREC assessors to be not relevant for the dataset's queries. The results in this column indicate that MORAG consistently reduces the number of nonrelevant documents retrieved, whereas the BOW fusion usually increases this number. This can be explained by the different ranking approach taken by a concept-based method: many nonrelevant documents retrieved by a keyword-based approach may include the query terms in a high frequency but are not related to the query. Other keyword-based systems, ranking by similar principles, are likely to rank these documents high as well and reinforce these false positives, whereas a conceptbased approach, ranking by conceptual similarity, is more likely to rank them low. This hypothesis is further reinforced by the "overlap of non-rel" column, where we explicitly quantify this overlap.

If we now revisit the Lemur fusion run, we'll notice that the two fused Lemur methods have not only a high overlap in relevant documents, but also a significantly high overlap in nonrelevant documents. Such a high overlap implies that nonrelevant documents are reinforced too, thus hurting the overall precision despite the substantial improvement in recall. This low result is despite the fact that the fused systems perform well individually and use quite different ranking approaches.

Finally, we point at a third group of documents worth examining: the unjudged documents. The "pooling" method used in the TREC methodology [Voorhees and Harman 1999] implies that only a small fraction of the corpus is evaluated for relevance by the human assessors, and any unjudged documents are then assumed nonrelevant. This approach was found to work well when comparing the relative performance of IR systems. However, research has shown that the use of pooling could discriminate against a new method that is based on novel principles [Zobel 1998], and it has been recommended that researchers consider the number of unjudged documents being fetched as an indication that performance is probably being underestimated. Following this recommendation, we found that our concept-based runs retrieved almost $40 \%$ more unjudged documents than an average BOW system (about 35000 documents compared to about 25000 in the evaluated BOW systems). Hence, there is reason to suspect that the true performance of MORAG may be even higher than the reported results, since some of these unjudged documents may well be relevant documents that could not be detected by any of the previous BOW approaches.

5.3.6. The Impact of Using More Relevant Examples. In this research, we have used the top and bottom ranked documents (in BOW retrieval) as positive and negative examples in the feature selection process. Naturally, these pseudo-relevant examples are a practical compromise, as they are assumed to be relevant (or nonrelevant) but may not be so in practice. Ideally, we would prefer to use only documents indicated as relevant or nonrelevant by the user. In considering this compromise, we were interested in learning more about the possible improvement to be gained by using better examples, and conducted additional experiments relying on TREC's human relevance judgments as "oracle" knowledge.

The retrieval process in these experiments was similar to that described in Section 4, except for the choosing of positive examples, for which we added a step 


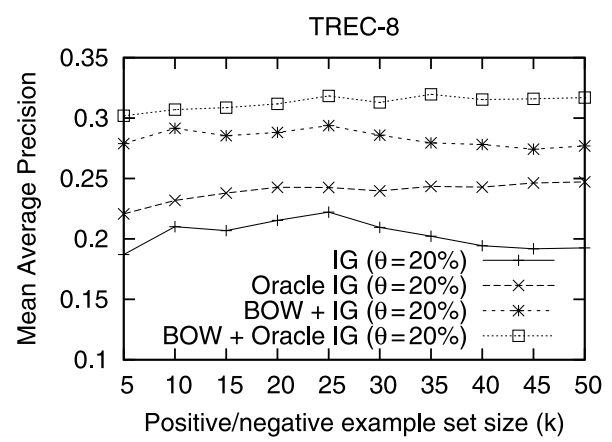

Fig. 22. Concept-based performance (IG FS) using pseudo-relevant examples versus true relevant examples.

of iterating through the top retrieved documents and selecting only those marked as relevant for this query in the TREC relevance judgments. Thus, the $k$ positive examples were chosen from a larger subset of top documents, and were guaranteed to be relevant. Negative examples are chosen as before, since relevant documents are very unlikely to appear in the bottom-ranked documents, and it is even less likely that the bottom-ranked documents will be judged at all. We then compare the results with those using standard pseudo-relevant positive examples.

Figure 22 shows results for the TREC-8 dataset using the IG FS method, with and without "oracle" relevance knowledge in choosing positive examples. The results indicate that using verified relevant documents as positive examples indeed improves performance by about 10\%-15\%. In addition, using more examples does not degrade performance as it did with pseudo-relevant examples (see, for example, Figure 12), reinforcing our earlier assumption that the decrease in performance was due to the decreasing relevance of lower-ranking documents. This result implies that there is value in more refined methods of choosing pseudo-relevant examples, which could be the subject of future work.

5.3.7. Estimating Optimal FS Performance. ESA-based performance was shown in Section 4.3 to depend directly on the choice of subset: a better selection process yielded better performance. It will be safe to assume that further research could derive even better FS methods than those described, and consequently better overall performance. We believe, therefore, that it would be worthwhile to estimate how much further improvement can be expected by employing MORAG with better feature selection methods.

In this final experiment, we iterated across all possible subsets of each query's initial features, and instead of using the described FS methods, we evaluated the subsets with relevance ("oracle") knowledge to find the one that gives optimal performance. Naturally, this process cannot be applied in a real-life scenario, but its results indicate the improvement that might be gained through better feature selection. Due to computation limitations, we only evaluated subsets of size $\leq 3$ out of initial 50 generated features, and subsets of size $\leq 4$ of initial 20 generated features.

Table V shows the results of these experiments. As expected, the performance of the resulting ESA queries was high, and at MAP of 0.3189 was even higher than the top keyword-based system we compared to (and far higher than the current optimal ESA-only result of 0.2223). Furthermore, fusing these results with BOW systems in MORAG yielded far better results, with improvements in performance of up to almost $50 \%$. 
Table V. TREC-8 Results with Several BOW Baselines, Using Optimal ("oracle") Concept Subset Selection

\begin{tabular}{cc|cc}
\hline $\begin{array}{c}\text { BOW } \\
\text { system }\end{array}$ & Baseline & $\begin{array}{c}\text { MorAG (optimal 4/20) } \\
(\mathrm{MAP}=0.2947)\end{array}$ & $\begin{array}{c}\text { MoRAG (optimal 3/50) } \\
(\mathrm{MAP}=0.3189)\end{array}$ \\
\hline Xapian & 0.2481 & $0.3322(+33.9 \%)$ & $0.3692(+48.8 \%)$ \\
Okapi & 0.2787 & $0.3406(+22.2 \%)$ & $0.3714(+33.3 \%)$ \\
AT\&T & 0.2853 & $0.3475(+21.8 \%)$ & $0.3673(+28.7 \%)$ \\
PIRCS & 0.3063 & $0.3568(+16.5 \%)$ & $0.3792(+23.8 \%)$ \\
\hline
\end{tabular}

All results were statistically significant.

In addition, comparing the performance of the two experiments (best 4 out of 20 initial features and best 3 out of 50) shows that selecting out of a larger pool of features worked better despite fewer features being selected. This result indicates that if superior feature selection capability is available, it would be preferable to select from a longer prefix of the ESA concept vector. Note that a result for subsets of size $\leq 4$ out of 20 features is an upper bound for subsets of size $\leq 3$ out of 20 , and thus this finding is valid even though the subset sizes were not identical. For some queries the optimal subset indeed was a smaller set, occasionally even a single feature, which indicates that using a uniform selection level parameter $(\theta)$ is not an optimal strategy. Future work may investigate methods that utilize a per-query selection level, possibly using ideas such as query clarity [Cronen-Townsend et al. 2002].

5.3.8. Performance Considerations. An important practical issue for any IR system is that of performance. The need to generate and select ESA features at query time may cause the system response time to become impractical, and the concepts index that stores concept vectors for documents and passages may incur heavy costs in storage requirements. We describe the performance measures of MORAG in our experimental implementation, which turned out to be reasonable even without undertaking any explicit performance-related optimizations.

The system was deployed on a single G5 PowerMac machine with four $2.5 \mathrm{GHz}$ cores and 12GB of RAM; in practice, the multiple cores were utilized only in indexing. In average, generating an ESA-based concept query out of the input text keywords took about 20-25ms. Most of this time was required for ESA feature generation, and not on the selection process. Retrieving results based solely on that concept query (Section 4) required an average of $200 \mathrm{~ms}$. Fetching results of the fused concept-based and BOW retrieval (Section 5) took, when ignoring the BOW subsystem time which is system dependent and can easily be parallelized, about $450 \mathrm{~ms}$. As for disk space, the entire ESA index required about 25GB for the 520K documents in TREC4-5, while the BOW index took about 16GB (sizes include indexing of both documents and passages). It should also be noted that we indexed concepts using their textual labels as tokens which is clearly inefficient; we kept these long labels for experimentation convenience.

\section{RELATED WORK}

Early approaches to concept-based IR attempted to leverage preexisting conceptual thesauri such as Wordnet [Miller et al. 1990] for concept representation. Wordnet's synsets, like ESA concepts, represent real-life semantic human concepts and provide an intuitive, natural representation. Unlike ESA, the mapping method was not automatically generated by leveraging an existing resource, but rather by manual assignment of terms to synsets by Wordnet's editors. For example, in the Estonia-related 
example query, Wordnet's editors provided the synonymous "Esthonia" form for "Estonia," and "economic system" or "thriftiness" equivalents for the different meanings of "economy." Using such synonyms may assist recall to some limited extent, but it is clear that the "thriftiness" sense is not the intended one for the query, and using it would cause the retrieval to drift away, degrading system performance. Hence, the success of synset-based retrieval depended on word sense disambiguation methods to select the correct synset in each occurrence. Previous research has shown inconsistent improvement with this approach [Sanderson 2000; Voorhees 1994], which was mostly found to be successful only when applied manually [Gonzalo et al. 1998] or augmented by other sources [Mandala et al. 1998; Stokoe et al. 2003].

A major drawback of manually mapping words to concepts is the great effort invested in achieving good coverage of the domain language. Some researchers chose to overcome this obstacle by turning to automatic construction of a thesaurus from the target corpus itself, somewhat similar to the automatic construction of an ESA model from an external knowledge base (e.g., Wikipedia). [Qiu and Frei 1993] described a method for extracting a similarity thesaurus based on cooccurrence in the target corpus, thus obtaining more relevant concepts based on implicit domain knowledge, and yielding effective improvement. Another variant method combining the two approaches was suggested in [Zhou et al. 2006], where a predefined dictionary of concepts was augmented with similar terms cooccurring in the corpus. Creating such cooccurrence resources is a computationally expensive process for large corpora, and one that needs to be constantly repeated for very dynamic corpora (such as the Web). With ESA-based concept representation the case is different, as the ESA feature generator is built once, regardless of the actual corpus used and of corpus changes.

Another automated approach used document ontologies as a source for concept representation. One example, KeyConcept [Gauch et al. 2003], is a retrieval system that maps documents to a limited subset of the concepts represented in the Open Directory Project ${ }^{9}$ (ODP), using documents categorized to those concepts as training data for concept classifiers, and conducting search on the augmented text/concept representation. The use of ODP as a source for concept representation and automatic mapping has some parallels with our ESA approach, in particular when considering that ESA was implemented over ODP data as well [Gabrilovich and Markovitch 2005]. However, the use of a limited concept ontology in KeyConcept resulted in a classifier that was not powerful enough to accurately classify the (short) queries. Thus query concepts were not automatically generated (as in this research) but had to be manually assigned by KeyConcept users. [Castells et al. 2007] describe another ontology-based approach, one that makes use of more formal semantic structures and queries and combines semantic search with keyword-based retrieval to compensate for the knowledge base incompleteness. As with KeyConcept, this article also assumes that semantic queries are created by the system user. The system was not evaluated on common IR benchmarks or against state-of-the-art IR systems.

Representing texts using concepts that are words, or explicit syntactic/semantic classes (such as Wordnet's synsets or ODP nodes), has the benefit of producing concepts that are human-readable, easy to analyze and reason about, and can be displayed to a user of such a system. ESA concepts, too, are based on human-defined natural concepts, as the example concept names throughout this article show. Yet concepts may also be defined using latent semantics, with possibly broader concept coverage. By analyzing the latent relationships between terms in the target corpus, methods such as Latent Semantic Indexing (LSI) [Deerwester et al. 1990] can project the term space

\footnotetext{
${ }^{9}$ http://dmoz.org
} 
to a reduced-dimensions concept space, shared by documents and queries, and thus be applied successfully to the IR task [Dumais 1994; Hofmann 1999].

Like generating an ESA model or a cooccurrence thesaurus, generating an LSI model for a large corpus involves heavy computation. Unlike ESA, though, the generated LSI model is corpus dependent, hence requiring the generation process to be repeated when the corpus changes or when a different corpus is used. In addition, beyond a certain size, LSI calculation becomes computationally nonfeasible, as it requires handling a full-scale term to document matrix, whereas ESA model generation has no such limitation and therefore can build a model with a very rich language coverage. Finally, the nonexplicit nature of resulting concepts makes LSI difficult to tune and reason about [Dumais 1994]. More recent dimensionality reduction methods applied to IR have included topic models approaches [Yi and Allan 2009] such as latent Dirichlet allocation [Wei and Croft 2006] and the Pachinko allocation model [Li and McCallum 2006].

All previously mentioned methods, including the one described in this article, apply concept-based analysis to both the indexing and the retrieval stages of IR. There also exists a large body of research applied to using concepts and ontologies in the retrieval stage only. Concept-based query expansion methods have been implemented using corpus-based methods [Grootjen and van der Weide 2006; Qiu and Frei 1993], domain-specific knowledge sources [Liu and Chu 2005], or an ontology derived from Web sources such as Wikipedia [Milne et al. 2007]. But methods based on query expansion, in addition to the aforementioned representation-related issues, are also vulnerable to expansion-specific problems such as query drift and sensitivity to parameter tuning [Billerbeck and Zobel 2004].

\section{CONCLUSION}

We have presented a novel approach to concept-based IR using ESA as a representation method, introducing a feature selection component that is based on pseudorelevance feedback. We have evaluated the proposed algorithms experimentally and demonstrated their improved performance. We have also estimated the potential for further improving the results of this approach, and outlined several insights in this regard that can guide future work.

Concept-based IR using ESA makes use of concepts that encompass human world knowledge, encoded into resources such as Wikipedia (from which an ESA model is generated), and that allow intuitive reasoning and analysis. Feature selection is applied to the query concepts to optimize the representation and remove noise and ambiguity. The results obtained by our proposed system (MORAG) are significantly better than the baselines used, including those of top performing systems in TREC-8. Analysis of the results shows that improving the performance of the FS component is possible and will directly lead to even better results. In future work we plan to optimize the documents' representation as well, by leveraging recent work on compact ESA representations [Liberman and Markovitch 2009].

We believe the results we have shown in Section 5.3, coupled with the potential improvement demonstrated there, position ESA and the MORAG framework as promising steps on the road to semantic retrieval solutions. Our work may provide both a leap in retrieval relevance and a potential shift in the IR paradigm, to one that is capable of manipulating human concepts rather than keywords only.

\section{ACKNOWLEDGMENTS}

The authors would like to thank Oren Kurland for his valuable comments on an earlier version of this article. 


\section{REFERENCES}

Allan, J., Callan, J., Feng, F.-F., And Malin, D. 1999. Inquery and trec-8. In Proceedings of the 8th Text Retrieval Conference (TREC-8). 637-644.

Anderka, M. And Stein, B. 2009. The esa retrieval model revisited. In Proceedings of the 32nd International ACM SIGIR Conference on Research and Development in Information Retrieval. ACM, 670-671.

ARAmpatzis, A. AND KAMPS, J. 2008. A study of query length. In Proceedings of the 31st Annual International ACM SIGIR Conference on Research and Development in Information Retrieval. ACM, 811-812.

Armstrong, T. G., Moffat, A., Webber, W., AND Zobel, J. 2009. Improvements that don't add up: Ad-Hoc retrieval results since 1998. In Proceeding of the 18th ACM Conference on Information and Knowledge Management (CIKM'09). ACM, 601-610.

Billerbeck, B. AND ZoBel, J. 2004. Questioning query expansion: An examination of behaviour and parameters. In Proceedings of the 15th Australasian Database Conference. Australian Computer Society, $69-76$.

BuCKLey, C. AND Robertson, S. 2008. Relevance feedback track overview: Trec 2008. In Proceedings of the 17th Text REtrieval Conference (TREC-17).

CALlan, J. P. 1994.Passage-Level evidence in document retrieval. In Proceedings of the 17th Annual International ACM SIGIR Conference on Research and Development in Information Retrieval. ACM/Springer, $302-310$

Castells, P., Fernandez, M., And Vallet, D. 2007. An adaptation of the vector-space model for ontologybased information retrieval.IEEE Trans. Knowl. Data Engin. 19, 2, 261-272.

Chang, M.-W., Ratinov, L., Roth, D., And SRikumar, V. 2008. Importance of semantic representation: Dataless classification. In Proceedings of the $23 r d$ AAAI Conference on Artificial Intelligence. AAAI Press, 830-835.

Croft, B. W. 2000. Combining Approaches to Information Retrieval. Kluwer Academic Publishers, Chapter $1,1-36$.

Cronen-Townsend, S., Zhou, Y., And Croft, W. B. 2002. Predicting query performance. In Proceedings of the 25th Annual International ACM SIGIR Conference on Research and Development in Information Retrieval. ACM, 299-306.

Deerwester, S. C., Dumais, S. T., Landauer, T. K., Furnas, G. W., and Harshman, R. A. 1990. Indexing by latent semantic analysis. J. Amer. Soc. Inf. Sci. 41, 6, 391-407.

Dumais, S. T. 1994. Latent semantic indexing (1si) and trec-2. In Proceedings of the 2nd Text Retrieval Conference (TREC-2). 105-116.

Egozi, O., Gabrilovich, E., AND Markovitch, S. 2008. Concept-Based feature generation and selection for information retrieval. In Proceedings of the $23 \mathrm{rd}$ AAAI Conference on Artificial Intelligence. AAAI Press, 1132-1137.

Fox, E. A. AND Shaw, J. A. 1994. Combination of multiple searches. In Proceedings of the 2nd Text REtrieval Conference (TREC-2). 243-252.

Fuller, M., Kaszkiel, M., Kimberley, S., NG, C., Wilkinson, R., Wu, M., And Zobel, J. 1999. The rmit/csiro ad hoc, q\&a, web, interactive, and speech experiments at trec 8. In Proceedings of the 8th Text REtrieval Conference (TREC-8). 549-564.

Furnas, G. W., Landauer, T. K., Gomez, L. M., And Dumais, S. 1987. The vocabulary problem in human-system communication. Comm. ACM 30, 11, 964-971.

GABrilovich, E. AND MARKOvitch, S. 2005. Feature generation for text categorization using world knowledge. In Proceedings of the 19th International Joint Conference on Artificial Intelligence (IJCAI'05). Morgan Kaufmann Publishers, 1048-1053.

GABRILOvich, E. AND MARKovitch, S. 2006. Overcoming the brittleness bottleneck using wikipedia: Enhancing text categorization with encyclopedic knowledge. In Proceedings of the 21st National Conference on Artificial Intelligence (AAAI'06). AAAI Press, 1301-1306.

Gabrilovich, E. AND MARKovitch, S. 2007. Computing semantic relatedness using wikipedia-based explicit semantic analysis. In Proceedings of the 20th International Joint Conference on Artificial Intelligence (IJCAI'07). Morgan Kaufmann Publishers, 1606-1611.

Gauch, S., Madrid, J. M., Induri, S., Ravindran, D., And Chadlavada, S. 2003. Keyconcept: A conceptual search engine. Tech. rep. TR-8646-37, University of Kansas.

Gonzalo, J., Verdejo, F., Chugur, I., AND CigarRin, J. 1998. Indexing with wordnet synsets can improve text retrieval. In Proceedings of the COLING/ACL Workshop on Usage of WordNet for NLP.

Grootjen, F. AND VAN DER WeIde, T. P. 2006. Conceptual query expansion. Data Knowl. Engin. 56, $174-193$.

ACM Transactions on Information Systems, Vol. 29, No. 2, Article 8, Publication date: April 2011. 
GUPTA, R. AND RATINOV, L.-A. 2008. Text categorization with knowledge transfer from heterogeneous data sources. In Proceedings of the 23rd AAAI Conference on Artificial Intelligence. AAAI Press, 842-847.

Gurevych, I., Muller, C., AND ZesCH, T. 2007. What to be? - Electronic career guidance based on semantic relatedness. In Proceedings of the Association for Computational Linguistics (ACL). The Association for Computer Linguistics, 1032-1039.

Guyon, I. AND Elisseeff, A. 2003. An introduction to variable and feature selection. J. Mach. Learn. Res. 3, 1157-1182.

HAW KING, D. 1999. Acsys trec-8 experiments. In Proceedings of the 8th Text REtrieval Conference (TREC-8). 307-316.

Hofmann, T. 1999. Probabilistic latent semantic indexing. In Proceedings of the 22nd Annual International ACM SIGIR Conference on Research and Development in Information Retrieval (SIGIR'99). ACM, $50-57$.

Huang, X., Huang, Y. R., Wen, M., An, A., LiU, Y., And Poon, J. 2006. Applying data mining to pseudorelevance feedback for high performance text retrieval. In Proceedings of the 6th IEEE International Conference on Data Mining (ICDM'06). IEEE Computer Society, 295-306.

John, G. H., KohAVI, R., AND PfLEGER, K. 1994. Irrelevant features and the subset selection problem. In Proceedings of the 11th International Conference on Machine Learning. 121-129.

KAPTEIN, R., KAMPS, J., AND HiEmstrA, D. 2008. The impact of positive, negative and topical relevance feedback. In Proceedings of the 17th Text REtrieval Conference (TREC-17).

KASZKiel, M. AND ZoBel, J. 2001. Effective ranking with arbitrary passages. J. Amer. Soc. Inf. Sci. Technol. 52, 4, 344-364.

Kwok, K. L., Grunfeld, L., AND Chan, M. 1999. Trec-8 ad-hoc, query and filtering track experiments using pircs. In Proceedings of the 8th Text Retrieval Conference (TREC-8). 217-228.

LEE, J. H. 1995. Combining multiple evidence from different properties of weighting schemes. In Proceedings of the 18th Annual International ACM SIGIR Conference on Research and Development in Information Retrieval. ACM, 180-188.

Li, W. ANd McCallum, A. 2006. Pachinko allocation: Dag-Structured mixture models of topic correlations. In Proceedings of the 23rd International Conference on Machine Learning (ICML'06). ACM, 577-584.

Liberman, S. AND MARKovitch, S. 2009. Compact hierarchical explicit semantic representation. In Proceedings of the IJCAI Workshop on User-Contributed Knowledge and Artificial Intelligence: An Evolving Synergy (WikiAI'09). Morgan Kaufmann Publishers.

LiU, X. AND CRoft, W. B. 2002. Passage retrieval based on language models. In Proceedings of the 11th International Conference on Information and Knowledge Management. ACM, 375-382.

LIU, Z. AND CHU, W. W. 2005. Knowledge-Based query expansion to support scenario-specific retrieval of medical free text. In Proceedings of the ACM Symposium on Applied Computing. ACM, 1076-1083.

Mandala, R., TAKenobu, T., AND Hozumi, T. 1998. The use of wordnet in information retrieval. In Proceedings of the COLING/ACL Workshop on Usage of WordNet in Natural Language Processing Systems. $31-37$.

Miller, G. A., Beckwith, R., Fellbaum, C., Gross, D., And Miller, K. J. 1990. Introduction to wordnet: An on-line lexical database. Int. J. Lexicograph. 3, 235-244.

Milne, D. N., Witten, I. H., AND Nichols, D. M. 2007. A knowledge-based search engine powered by wikipedia. In Proceedings of the 16th ACM Conference on Information and Knowledge Management. ACM, 445-454.

Mitra, M., Singhal, A., AND Buckley, C. 1998. Improving automatic query expansion. In Proceedings of the 21st Annual International ACM SIGIR Conference on Research and Development in Information Retrieval. ACM, 206-214.

Ozcan, R. And Aslandogan, Y. A. 2005. Concept-based information access. In Proceedings of the International Conference on Information Technology: Coding and Computing (ITCC'05). IEEE Computer Society, 794-799.

Potthast, M., Stein, B., AND AnderkA, M. 2008. A wikipedia-based multilingual retrieval model. In Proceedings of the 30th European Conference on IR Research (ECIR). Springer, 522-530.

QIU, Y. AND FREI, H. P. 1993. Concept based query expansion. In Proceedings of the 16th Annual International ACM SIGIR Conference on Research and Development in Information Retrieval. ACM Press, 160-169.

QUiNLAN, J. R. 1986. Induction of decision trees. Mach. Learn. 1, 1, 81-106.

Robertson, S. E. AND WALKer, S. 1999. Okapi/keenbow at trec-8. In Proceedings of the 8th Text REtrieval Conference (TREC-8). 151-162. 
Rocchio, J. J. 1971. Relevance feedback in information retrieval. In The SMART Retrieval System: Experiments in Automatic Document Processing, G. Salton Ed., Prentice Hall, Englewood Cliffs, NJ, 313-323.

Ruthven, I. AND Lalmas, M. 2003. A survey on the use of relevance feedback for information access systems. Knowl. Engin. Rev. 18, 2, 95-145.

SAlton, G. AND BUCKLEY, C. 1990. Improving retrieval performance by relevance feedback. J. Amer. Soc. Inf. Sci. 41, 4, 288-297.

SANDERSON, M. 2000. Retrieving with good sense. Inf. Retriev. 2, 1, 49-69.

Schuetze, H. AND Pedersen, J. O. 1995. Information retrieval based on word senses. In Proceedings of the 4th Annual Symposium on Document Analysis and Information Retrieval. 161-175.

Singhal, A., Abney, S., Bacchiani, M., Collins, M., Hindle, D., And Pereira, F. 1999. At\&t at trec-8. In Proceedings of the 8th Text REtrieval Conference (TREC-8). 317-330.

Singhal, A., Mitra, M., AND BuCKLEY, C. 1997. Learning routing queries in a query zone. In Proceedings of the 20th Annual International ACM SIGIR Conference on Research and Development in Information Retrieval. ACM, 25-32.

Singhal, A., Salton, G., Mitra, M., And Buckley, C. 1995. Document length normalization. Tech. rep. TR95-1529, Cornell University, Ithaca, NY.

Sorg, P. AND Cimiano, P. 2008. Cross-lingual information retrieval with explicit semantic analysis. In Working Notes for the CLEF 2008 Workshop.

Stokoe, C. P., OAKES, M., AND TAIT, J. 2003. Word sense disambiguation in information retrieval revisited. In Proceedings of the 26th Annual International ACM SIGIR Conference on Research and Development in Informaion Retrieval. ACM, 159-166.

Styltsvig, H. B. 2006. Ontology-based information retrieval. Ph.D. thesis, Department of Computer Science, Roskilde University, Denmark.

Vogt, C. C. And Cottrell, G. W. 1999. Fusion via a linear combination of scores. Inf. Retriev. 1, 3, $151-173$

VooRhees, E. M. 1993. Using wordnet to disambiguate word senses for text retrieval. In Proceedings of the 16th Annual International ACM SIGIR Conference on Research and Development in Information Retrieval. ACM, 171-180.

Voorhees, E. M. 1994. Query expansion using lexical-semantic relations. In Proceedings of the 17th Annual International ACM SIGIR Conference on Research and Development in Information Retrieval. Springer, 61-69.

VooRHeEs, E. M. 2005. Overview of the TREC 2004 robust retrieval track. In Proceedings of the 13th Text REtrieval Conference (TREC-13). 70-79.

VoORHEES, E. M. AND HARMAN, D. 1998. Overview of the seventh text retrieval conference (trec-7). In Proceedings of the 7th Text REtrieval Conference (TREC-7). 1-24.

VoorheEs, E. M. AND HARMAn, D. 1999. Overview of the eighth text retrieval conference (trec-8). In Proceedings of the 8th Text REtrieval Conference (TREC-8). 1-24.

Wei, X. AND Croft, W. B. 2006. Lda-Based document models for ad-hoc retrieval. In Proceedings of the 29th Annual International ACM SIGIR Conference on Research and Development in Information Retrieval (SIGIR'06). ACM, 178-185.

$\mathrm{XU}$, J. AND CROFT, W. B. 2000. Improving the effectiveness of information retrieval with local context analysis. ACM Trans. Inf. Syst. 18, 1, 79-112.

YAN, R., HAUPTMANN, A. G., AND Jin, R. 2003. Negative pseudo-relevance feedback in content-based video retrieval. In Proceedings of the 11th ACM International Conference on Multimedia. ACM, 343-346.

YANG, Y. AND PEDERSEN, J. O. 1997. A comparative study on feature selection in text categorization. In Proceedings of the 14th International Conference on Machine Learning. Morgan Kaufmann, 412-420.

YI, X. AND Allan, J. 2009. A comparative study of utilizing topic models for information retrieval. In Proceedings of the 31st European Conference on IR Research (ECIR). Springer, 29-41.

ZHOU, X., ZHANG, X., AND HU, X. 2006. Using concept-based indexing to improve language modeling approach to genomic ir. In Lecture Notes in Computer Science. Springer, 444-455.

ZoBel, J. 1998. How reliable are the results of large-scale information retrieval experiments? In Proceedings of the 21st Annual International ACM SIGIR Conference on Research and Development in Information Retrieval. ACM Press, 307-314.

Received February 2010; revised October 2010; accepted January 2011 